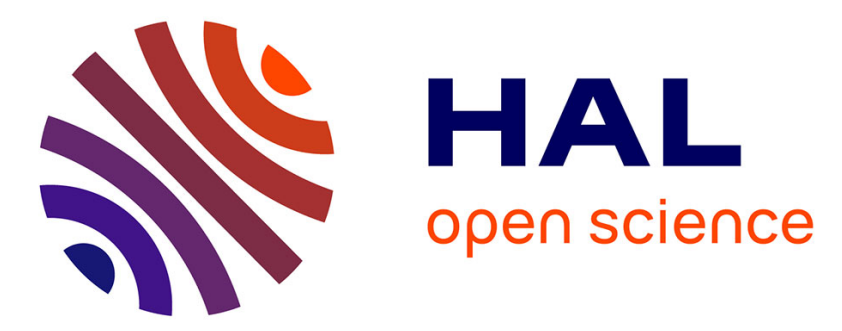

\title{
Évolutions récentes des émissions de CO2 liées à la mobilité des Français : analyser les dynamiques à l'œuvre grâce aux enquêtes nationales Transports de 1994 et 2008
}

\author{
Jean-Pierre Nicolas, Damien Verry, Zahia Longuar
}

\section{To cite this version:}

Jean-Pierre Nicolas, Damien Verry, Zahia Longuar. Évolutions récentes des émissions de CO2 liées à la mobilité des Français: analyser les dynamiques à l'œuvre grâce aux enquêtes nationales Transports de 1994 et 2008. Economie et Statistique / Economics and Statistics, 2012, 457-458, pp.161-183. halshs-01716169

\author{
HAL Id: halshs-01716169 \\ https://shs.hal.science/halshs-01716169
}

Submitted on 23 Feb 2018

HAL is a multi-disciplinary open access archive for the deposit and dissemination of scientific research documents, whether they are published or not. The documents may come from teaching and research institutions in France or abroad, or from public or private research centers.
L'archive ouverte pluridisciplinaire HAL, est destinée au dépôt et à la diffusion de documents scientifiques de niveau recherche, publiés ou non, émanant des établissements d'enseignement et de recherche français ou étrangers, des laboratoires publics ou privés. 


\title{
Évolutions récentes des émissions de $\mathrm{CO}_{2}$ liées à la mobilité des Français : analyser les dynamiques à l'œuvre grâce aux enquêtes nationales Transports de 1994 et 2008
}

\author{
Jean-Pierre Nicolas *, Damien Verry ** et Zahia Longuar *
}

Rappel :

Les jugements et opinions exprimés par les auteurs n'engagent qu'eux mêmes, et non les institutions auxquelles ils appartiennent, ni a fortiori l'Insee.
Les transports constituent une source importante d'émissions humaines de $\mathrm{CO}_{2}$. Centré sur les mobilités individuelles, cet article vise à appréhender les facteurs socioéconomiques explicatifs des émissions de $\mathrm{CO}_{2}$ résultant de la mobilité des personnes, ainsi que leurs évolutions au cours des dernières années. Il s'appuie sur les deux dernières enquêtes nationales Transports de 1994 et 2008. Ces enquêtes fournissent une image de l'ensemble des déplacements des personnes résidant sur le territoire national, qu'ils soient réalisés localement ou à longue distance et à l'étranger. Pour les besoins de cet article, les émissions de chaque déplacement recueilli par ces enquêtes ont été estimées, ce qui a permis de déterminer les facteurs explicatifs et les dynamiques à l'œuvre derrière les résultats agrégés à un grand niveau de détail.

Si les émissions progressent de $14 \%$ entre 1994 et 2008, les tendances ne sont pas les mêmes sur les territoires du quotidien qu'en longue distance. En ce qui concerne les déplacements locaux, la progression très nette $(+17 \%)$ est tirée notamment par la poursuite de l'étalement urbain alors que les habitants des espaces centraux diminuent leurs mobilités et leurs émissions. Les facteurs de cette augmentation résident essentiellement dans les contraintes liées aux déplacements domicile-travail des actifs, ainsi que dans des changements générationnels tels que la forte augmentation des effectifs des seniors et leur propension plus forte que celle de la génération précédente à utiliser la voiture. Sur la longue distance, l'augmentation du nombre de déplacements enregistrée sur la période est compensée en partie par une baisse des émissions unitaires, et la croissance des émissions de $\mathrm{CO}_{2}(+8 \%)$ reflète principalement la croissance de la population $(+6 \%)$.

\footnotetext{
* Laboratoire d'Économie des Transports (LET), ENTPE, Université de Lyon, rue M.Audin, 69518 Vaulx-en-Velin, Francefax : 04720470 92, email : nicolas@entpe.fr.

** Centre d'Études sur les Réseaux, les Transports, I'Urbanisme et les constructions publiques (Certu), 9, rue Juliette Récamier 69456 Lyon Cedex 06.
} 
$\mathbf{L}$ e protocole de Kyoto au niveau international, l'engagement européen du « facteur 4 » ou le Grenelle de l'environnement en France sont autant d'actions qui témoignent d'une volonté croissante, de la part des États, de limiter les émissions de dioxyde de carbone $\left(\mathrm{CO}_{2}\right)$ et l'impact des activités humaines sur l'effet de serre. Or, la mobilité des personnes et des biens est l'une des plus importantes sources d'émissions de $\mathrm{CO}_{2}$. Ainsi en 2004, le secteur des transports était responsable de $23 \%$ environ des émissions humaines de gaz à effet de serre au niveau mondial (GIEC, 2007). En France, du fait notamment d'une forte proportion d'énergie fournie par la filière nucléaire dans de nombreux secteurs, la part des transports circulant sur le territoire national, hors trafic aérien à plus de 1000 mètres d'altitude et trafic maritime international, s'élevait cette même année à $26 \%$ des émissions de gaz à effet de serre (répartis en $2 / 3$ liés aux mobilités individuelles et $1 / 3$ aux trafics de marchandises, Centre interprofessionnel technique d'études de la pollution atmosphérique (Citepa), 2010). Et, ce qui est plus préoccupant encore, la tendance a toujours été à la croissance dans ce secteur d'activité, mouvement qui se poursuit encore au niveau mondial, même si des tassements sont observés dans les pays occidentaux comme la France depuis le début des années 2000, en conséquence de la forte hausse des prix du pétrole et des carburants (Citepa, 2010 ; Besson, 2008).

Des bilans nationaux et internationaux d'émissions de $\mathrm{CO}_{2}$ établis à partir des trafics globaux permettent aujourd'hui d'avoir une vision d'ensemble cohérente et convergente de ces évolutions et des enjeux qu'elles représentent (Citepa, 2010 ; IEA, 2010 ; GIEC, 2007), même si des incertitudes existent et existeront toujours sur la mesure des trafics, de leurs émissions et des impacts induits (Fuglestvedt et al., 2010).

Ces bilans ne permettent cependant pas de faire le lien entre émission et émetteur, et donc de s'interroger, à un niveau fin, sur les types de personnes et/ou d'acteurs économiques à l'origine des émissions enregistrées et sur les logiques socioéconomiques susceptibles de les expliquer. Pour mieux répondre à ces questions qui constituent une autre facette de la problématique de la réduction des émissions de gaz à effet de serre imputables aux transports, une série de travaux concernant les mobilités individuelles a été développée en France à l'aide des enquêtes ménages déplacements (EMD). En effet, celles-ci permettent de relier les caractéristiques socioéconomiques des individus à leurs comportements de mobilité, à partir desquels des estimations des émissions peuvent être réalisées. On obtient alors une image des mobilités générées par les habitants d'un territoire permettant de comprendre les mécanismes socioéconomiques qui les motivent et une vision fine des émissions qu'elles entrainent. Ces travaux ont été essentiellement réalisés à partir des EMD locales. Initiés par Orfeuil (1984) pour établir le bilan des consommations énergétiques dues aux mobilités au sein d'une agglomération, ils ont été progressivement élargis aux émissions de polluants atmosphériques (Gallez, 1995 ; Gallez et Hivert, 1998), puis ouverts à d'autres thématiques comme celles des coûts (Nicolas et al., 2002 ; Vanco, 2011), et des travaux se poursuivent pour intégrer ces résultats dans des bilans exhaustifs sur une aire urbaine complète (Hivert et al., 2009).

Par contre, ces enquêtes locales ne concernent que les mobilités quotidiennes locales des personnes résidant à l'intérieur des périmètres enquêtés. Les déplacements à longue distance et de week-end ainsi que les mobilités des personnes vivant en dehors des grandes agglomérations enquêtées ne sont pas pris en compte. Ainsi, l'analyse des émissions globales liées aux mobilités individuelles est-elle incomplète en raison de l'absence de telles données. Les Enquêtes Nationales Transports (ENT) menées tous les 10-15 ans au niveau national peuvent aider à pallier cette lacune car elles visent à fournir une image globale des mobilités individuelles et portent sur les résidents de l'ensemble du territoire français. Un premier travail a déjà été mené dans ce sens à partir de l'enquête nationale Transports de 1994 (Nicolas et David, 2009). La réalisation d'une nouvelle enquête du même type en 2008 permet de mettre à jour cette image et d'analyser les évolutions au cours de ces 15 années qui ont vu croître fortement les préoccupations en matière d'effet de serre.

Ainsi est-il désormais possible d'avancer quelques éléments permettant d'appréhender les facteurs socioéconomiques individuels explicatifs des niveaux de $\mathrm{CO}_{2}$ générés par les mobilités des personnes et de comprendre les évolutions enregistrées au cours des dernières années. Ces analyses sont d'abord données au niveau global, puis détaillées en distinguant les déplacements locaux et les déplacements à longue distance. On donne dans un premier temps les grandes lignes de la méthode utilisée. 


\section{Du calcul des émissions par déplacement à l'analyse des évolutions par sous-groupes de population}

$\mathbf{L}$ 'estimation des émissions de $\mathrm{CO}_{2}$ s'appuie sur les enquêtes nationales Transport de 1994 et de 2008. Ces enquêtes fournissent un panorama de la mobilité quotidienne et de la mobilité sur longue distance des personnes résidentes sur le territoire français. Elles distinguent les déplacements locaux « de la veille et du week-end » des déplacements sur longue distance ( au cours des trois derniers mois »), et comportent leur description détaillée ainsi que celle des véhicules qu'ils mettent en jeu, afin de permettre une estimation des émissions de $\mathrm{CO}_{2}$ à ce niveau élémentaire du déplacement (cf. encadré 1). Ainsi est-il possible, en partant de ce niveau fin (le déplacement), d'effectuer une estimation des émissions de $\mathrm{CO}_{2}$ avec une grande souplesse quant au choix du niveau d'analyse : ce dernier est en effet obtenu comme une agrégation des niveaux élémentaires (cf. encadré 2). Il est alors possible de déterminer les facteurs explicatifs et les dynamiques à l'œuvre derrière les résultats agrégés à un grand niveau de détail.

Encadré 1

\section{LES ENQUÊTES NATIONALES TRANSPORTS DE 1994 ET DE 2008}

Depuis la fin des années 1950, on effectue tous les dix ans environ une enquête nationale dégageant un panorama de la mobilité quotidienne et à longue distance des personnes résidantes sur le territoire français (Madre, 1997). Les trois dernières campagnes ont été menées en collaboration étroite entre l'Insee et l'Institut national de recherche sur les transports et leur sécurité (Inrets), en 1981/82, 1993/94 et 2007/08. Elles fournissent une base cohérente et exhaustive pour l'analyse des facteurs socioéconomiques qui influent sur les mobilités individuelles.

Les enquêtes nationales Transports apportent plusieurs éclairages sur la mobilité des Français (Armoogum et al., 2010). Les informations recueillies sont regroupées dans plusieurs fichiers de natures différentes. Parmi les éléments qui intéressent plus particulièrement notre étude, on mentionnera :

- un carnet de bord, qui recueille tous les déplacements d'une voiture du ménage pendant une semaine ;
- un questionnaire recueillant les déplacements de la veille et du dernier week-end, auprès d'une personne tirée au sort parmi les individus du ménage de 6 ans ou plus présents au moment de l'enquête ;

- un questionnaire recueillant de manière spécifique les déplacements réalisés à longue distance (ie à plus de $80 \mathrm{~km}$ à vol d'oiseau du domicile) au cours des trois derniers mois précédents l'enquête. Dans l'enquête de 1994, deux tirages au sort séparés avaient été effectués au sein du ménage pour chacun des deux questionnaires, et donc seul un nombre limité d'individus (7 729) ont été interrogés à la fois sur leur mobilité de la veille et sur leur mobilité à longue distance. En 2008, les deux questionnaires ont été administrés à l'ensemble des individus.

Quelques éléments de comparaison entre les enquêtes de 1994 et 2008 sont donnés par le tableau suivant :

\section{Comparaison des enquêtes nationales Transports de 1994 et 2008}

\begin{tabular}{|l|c|c|}
\hline & 1994 & 2008 \\
\hline Nombre de ménages enquêtés & 14213 & 20178 \\
\hline Déplacements recueillis en mobilité de la veille et du week-end & 92925 & 126476 \\
\hline Déplacements recueillis en mobilité longue distance & 41774 & 18718 \\
\hline
\end{tabular}

Tous les déplacements, à motifs privés comme dans le cadre du travail, sont pris en compte. Seuls les déplacements professionnels des personnes dont le métier est de se déplacer (chauffeurs routiers, livreurs, taxis, etc.) sont omis. Les caractéristiques socioéconomiques du ménage et des personnes interrogées sont par ailleurs saisies lors de l'enquête. De même, une description détaillée des véhicules du ménage est recueillie.

Compte tenu de notre problématique d'estimation des émissions de $\mathrm{CO}_{2}$ et d'analyse des facteurs explicatifs de leur genèse et de leurs évolutions, presque tous ces éléments de l'enquête sont exploités. Les informations fournies par le questionnaire spécifique sur les déplacements à longue distance complètent celles issues des déplacements de la veille, permettant de disposer d'une image complète de la mobilité des personnes résidant sur le territoire français. Leurs caractéristiques socioéconomiques constituent la toile de fond analytique des comportements de déplacements. Enfin, la description détaillée des véhicules et des déplacements permet un calcul plus précis des émissions de $\mathrm{CO}_{2}$ (cf. encadré 2). 


\section{Les facteurs explicatifs des évolutions des émissions}

Une fois les émissions calculées pour chaque déplacement, des agrégations peuvent être réalisées au niveau global comme par sousgroupe d'individus ou par mode par exemple.

Le volume global des émissions d'une année $t, \mathrm{E}_{t}$, peut être exprimé comme le produit de la distance moyenne parcourue par personne et par an $\left(\mathrm{d}_{t}\right.$, en $\mathrm{km} /$ pers/an), multipliée par le niveau moyen d'émission par unité de distance $\left(\mathrm{eu}_{t}\right.$ en $\mathrm{g} / \mathrm{km}$ ) et par la population totale $\left(\mathrm{p}_{t}\right.$ en nombre de personnes), soit: $\mathrm{E}_{t}=\mathrm{p}_{t} \cdot \mathrm{d}_{t} \cdot \mathrm{eu}_{t}$

Si l'on s'intéresse à des évolutions comme c'est le cas dans cet article, le niveau global d'émissions $\mathrm{E}_{t+1}$ de la date $t+1$ peut être déduit de $\mathrm{E}_{t}$ et des taux d'évolution de la population, de la

\section{Encadré 2}

\section{ESTIMATION DES ÉMISSIONS DE $\mathrm{CO}_{2}$ À PARTIR DES ENQUÊTES NATIONALES TRANSPORTS}

Chaque déplacement enregistré dans l'enquête est caractérisé par plusieurs variables qui rendent possible une estimation de ses émissions (cf. schéma ci-dessous). Partir de ce niveau élémentaire du déplacement autorise une très grande souplesse dans les analyses ultérieures. Les résultats peuvent en effet être ensuite agrégés au niveau d'analyse désiré, par mode, par type de déplacement ou, en ce qui concerne cette étude, par type de personne.

Les calculs d'émission de $\mathrm{CO}_{2}$ réalisés reposent sur la méthodologie proposée dans le programme européen COPERT 4 (COmputer Program to calculate Emissions from Road Transport; Ntziachristos et al., 2009), complété par des données émanant du rapport Deloitte (2008) et des travaux du programme MEET « Methodology for calculating transport emissions and energy consumption " (European Communities, 2000). Les grands principes de calcul retenus dans ce travail sont présentés ci-dessous et on trouvera le détail des choix et hypothèses dans Longuar et al. (2012), avec des précisions dans Raux et al. (2005) pour certains traitements spécifiques à l'enquête de 1994.

Les bases utilisées pour estimer les émissions de $\mathrm{CO}_{2}$ à partir des enquêtes nationales Transports de 1994 et 2008

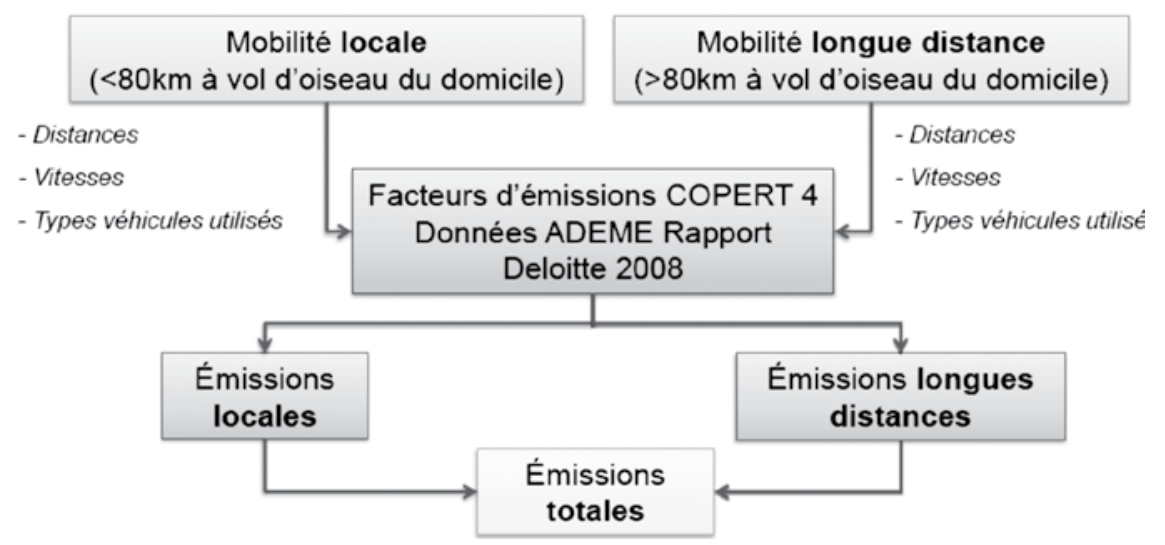

Les principes retenus pour les différents modes de transport peuvent être présentés rapidement.

La Voiture Particulière. Mobilités locale et sur longue distance confondues, l'automobile est le mode de transport prédominant puisqu'elle représente $72,9 \%$ du total des distances parcourues en 1994 et $71,8 \%$ en 2008. Pour ce mode, COPERT 4 fournit des courbes d'émissions en fonction de la vitesse moyenne sur le parcours, de la cylindrée, de la norme d'émissions respectée et du type de carburation des véhicules. Les enquêtes nationales Transports fournissent directement l'information sur cette dernière caractéristique. La norme est déduite de l'âge du véhicule au moment de l'enquête. Par ailleurs, la cylindrée peut être inférée en croisant les informations fournies par l'enquête sur la puissance fiscale, l'âge et la carburation des véhicules (Gallez et Hivert, 1998).

Pour chaque déplacement, la distance parcourue sur les différents réseaux (urbain, routier, autoroutier) a pu être établie. Elle était directement recueillie en 1994, elle a été recalculée à partir d'une estimation du système d'information géographique (SIG) pour 2008. Une vitesse moyenne a été fixée pour chacun de ces réseaux à partir du distancier Viamichelin, ce qui a permis ensuite de calculer les émissions à partir de COPERT. Les tests que nous avons réalisés montrent qu'aux niveaux agrégés 
distance moyenne par personne et des émissions unitaires, notés respectivement $\mathrm{r}_{p}, \mathrm{r}_{d}$ et $\mathrm{r}_{e u}$ :

$\mathrm{E}_{t+1}=\mathrm{E}_{t} \cdot\left(1+\mathrm{r}_{E}\right)=\mathrm{E}_{t} \cdot\left[\left(1+\mathrm{r}_{p}\right) \cdot\left(1+\mathrm{r}_{d}\right) \cdot\left(1+\mathrm{r}_{e u}\right)\right]$

Ces équations peuvent ensuite être enrichies pour mieux prendre en compte le détail des comportements et des répartitions modales, mais le principe de décomposition reste le même.

Chaque mode se caractérise par une quantité d'émission et une évolution de cette dernière au cours des quinze années étudiées bien distinctes. Il répond également à des besoins

Encadré 2 (suite)

de nos analyses, ces différences méthodologiques entre les enquêtes de 1994 et 2008 n'induisent pas de variations significatives dans les estimations d'émissions de $\mathrm{CO}_{2}$ (Longuar et al., 2012).

Enfin, les émissions de $\mathrm{CO}_{2}$ ainsi établies ont été attribuées à la personne enquêtée au prorata du nombre d'occupants du véhicule, qui est également appréhendé par l'enquête.

Les autres modes apparaissent plus marginaux en termes de distances parcourues : I'avion (10,7\% en 1994, 12,0 \% en 2008), le train (respectivement 5,2 et $5,6 \%$ ), puis les transports collectifs urbains (5,5 et $5,9 \%)$, les modes doux $(1,7 \%$ aux deux dates) et tous les autres modes (3,5 et $3,0 \%)$. Des émissions unitaires ont été établies pour chacun de ces modes à partir de la méthodologie proposée par MEET pour l'enquête de 1994 (EC, 1999), et des résultats du rapport Deloitte (2008) pour l'enquête 2008. Là encore, les distances parcourues ont été estimées à partir des origines et destinations des déplacements et à l'aide des outils SIG. Dans le cas de l'avion, l'application de tels coefficients moyens indépendants du déplacement fournit un résultat correct au niveau global, mais conduit à lisser les variations qui peuvent être très fortes suivant la longueur du déplacement (en raison de la forte consommation de carburant aux moments du décollage et de l'atterrissage qui a pour corollaire que ces deux épisodes du vol pèsent beaucoup en termes d'émission de $\mathrm{CO}_{2}$ ), le type d'aéronef et son taux de remplissage.

Le tableau ci-dessous donne un ordre de grandeur des émissions moyennes de $\mathrm{CO}_{2}$ obtenues au terme de ces calculs par voyageur.kilomètre (Longuar et al., 2010), compte tenu des émissions de chaque mode et des taux de remplissage moyens correspondants.

\section{Émissions moyennes de $\mathrm{CO}_{2}$ des différents modes (g/pers.km)}

\begin{tabular}{|l|r|r|}
\hline \multirow{2}{*}{} & \multicolumn{2}{|c|}{ Émissions unitaires } \\
\cline { 2 - 3 } & 1994 & 2008 \\
\hline Déplacements locaux & & 173 \\
Automobile & 165 & 42 \\
Transports collectifs urbains & 55 & 0 \\
Modes doux & 0 & 103 \\
\hline Déplacements sur longue distance & & 10 \\
Automobile & 98 & 128 \\
Train & 11 & 169 \\
Avion & 169 & \multicolumn{2}{|c|}{} \\
\hline
\end{tabular}

Lecture : en 1994, l'émission unitaire moyenne d'un passager automobile est de 165 grammes de $\mathrm{CO}_{2}$ par kilomètre parcouru pour ses déplacements locaux; elle est de 98 grammes pour ses déplacements sur longue distance.

Champ : individus âgés de 6 ans ou plus résidant en France métropolitaine ; émissions calculées avec le taux de remplissage déclaré, enfants de moins de 6 ans inclus.

Source : traitement LET-Certu à partir de Insee-Inrets : enquête nationale Transports 1994, et de Insee, Inrets, SoeS : enquête nationale Transports et déplacements 2008.

Les émissions calculées dans le cadre de cet article sont établies « du réservoir à la roue » : elles prennent en compte uniquement la consommation d'énergie directe et font abstraction de l'énergie primaire utilisée (par exemple, l'énergie thermique pour produire l'électricité s'il s'agit d'un mode de transport utilisant l'électricité : tramway, train électrique, etc.) ainsi que les émissions liées à la production et au transport des carburants (raffinage, distribution...). Enfin, l'enquête nationale Transports rend compte de l'ensemble des déplacements des personnes résidant en France métropolitaine. C'est donc un critère résidentiel et non territorial qui s'applique. Ainsi, comme nous l'avons évoqué en introduction, deux grandes différences peuvent être relevées par rapport aux inventaires officiels produits par le Citépa. D'une part, on estime les émissions des résidents du territoire français, quelle que soit leur nationalité, y compris lorsqu'ils vont à l'étranger, et on ne mesure pas les émissions que les non-résidents réalisent sur le sol français. D'autre part, les déplacements réalisés en avion sont pris en compte sur l'ensemble de leur trajet, et pas seulement sur les premiers mille mètres d'altitude au décollage et à l'atterrissage comme dans le cas des inventaires territoriaux. 
particuliers, tant en termes de distances à parcourir que de milieu où se réalisent les déplacements. L'intensité de l'usage de ces modes, exprimée en kilomètre parcourus par personne et par an, constitue ainsi un marqueur des modes de vie et des mobilités qu'ils entraînent, même si elle ne peut, à l'évidence, prétendre embrasser toutes les dimensions de la mobilité (Gallez et Orfeuil, 1998). Saisir les variations d'émissions de $\mathrm{CO}_{2}$ exige donc de répartir les différents modes entre quelques catégories homogènes: automobile, transports collectifs, modes doux (marche à pied et vélo) et autres modes pour ce qui est des déplacements au niveau local, et automobile, train, avion et autres modes pour les déplacements sur longue distance.

Par ailleurs, deux typologies des individus ont été réalisées pour prendre en compte les différences de mobilité (cf. parties 1.4. et 1.5 suivantes). Elles ont été construites en cherchant à constituer des groupes aux comportements les plus homogènes possibles, tout en conservant un schéma explicatif cohérent des mobilités. Les évolutions démographiques au sein de chacun des groupes peuvent ainsi être observées, de même que leurs changements de comportement.

Ainsi, cette double distinction entre les modes et les segments de population aux mobilités différenciées, a été opérée en ramenant les comportements de mobilité à des distances totales parcourues par mode et par personne à l'intérieur de chaque groupe, exprimées par le produit du nombre annuel de déplacements selon le mode par la distance moyenne d'un déplacement du même mode. L'équation initiale peut alors être détaillée de la manière suivante :

$E_{t}=\Sigma_{\mathrm{i}} \operatorname{pop}_{i} \cdot \Sigma_{\mathrm{i} j \mathrm{j}}\left(\operatorname{dep}_{i}^{j} \cdot \operatorname{dist}_{i}^{j} \cdot e u_{i}^{j}\right)$

avec $E_{t}$ les volumes d'émissions de $\mathrm{CO}_{2}$ en $t$, pop $_{i}$ la population totale du groupe i à la date $\mathrm{t}$, $d e p_{i}^{j}$ le nombre quotidien moyen de déplacements des personnes du groupe $\mathrm{i}$ avec le mode $\mathrm{j}$, dist $_{i}^{j}$ la distance moyenne d'un déplacement en mode $\mathrm{j}$ des personnes du groupe i et $e u_{i}^{j}$ les émissions unitaires moyennes du mode $\mathrm{j}$ pour les personnes du groupe $\mathrm{i}$.

Les taux de variations de ces facteurs seront respectivement notés rpop ${ }_{i}, \operatorname{rdep}_{\mathrm{i}}^{\mathrm{j}}$, $\operatorname{rdist}_{\mathrm{i}}^{\mathrm{j}}$, et reu $\mathrm{re}_{\mathrm{i}}^{\mathrm{j}}$. Ils rendent compte des dynamiques à l'intérieur de chaque groupe et permettent de reconstituer les évolutions globales.

\section{La mobilité locale et la mobilité sur longue distance répondent à des logiques différentes}

Si l'on se réfère à la littérature, la mobilité locale correspond d'abord à une mobilité du quotidien, où les déplacements contraints pour aller travailler ou étudier sont prévalants et structurants (Orfeuil, 2002). Les enquêtes ménages sur les déplacements locaux montrent bien que même s'ils diminuent en nombre, ces déplacements contraints restent prédominants en termes de distance parcourue du fait de leur longueur moyenne; ils sont également structurants dans la mesure où ils entraînent des contraintes temporelles fortes et déterminent par là même l'organisation des autres activités quotidiennes. La croissance démographique des seniors, qui ne sont pas soumis à ces activités contraintes, n'a pas suffi à contrebalancer cette tendance lourde qui a porté l'essentiel de la croissance des distances parcourues en mobilité locale entre 1994 et 2008 (Quételard, 2010). À l'inverse, la mobilité sur longue distance est occasionnelle, et correspond beaucoup plus à des déplacements non obligés, tels que les vacances ou des visites à des amis ou de la famille. La contrainte budgétaire est sensible pour cette mobilité, aussi le niveau de revenu constitue-t-il le premier facteur de clivage (Paulo, 2006 ; Grimal, 2010).

Dans les enquêtes nationales Transports, la distinction entre mobilité locale et mobilité sur longue distance s'opère sur un critère de distance de 80 kilomètres du domicile. À l'intérieur de ce périmètre, les déplacements sont considérés comme relevant du local ; au-delà, ils relèvent de la longue distance. Malgré la part d'arbitraire de ce type de définition, les logiques de mobilité qu'elle permet de distinguer sont suffisamment différentes pour la justifier. Ainsi, selon les résultats de l'enquête de 1994, les déplacements sur longue distance sont réalisés à $80 \%$ pour des motifs personnels. Leur fréquence annuelle (12,6 par an et par personne) est relativement peu élevée, même s'ils totalisent en moyenne $5230 \mathrm{~km}$ par an et par personne. La mobilité locale, quand à elle, représente en moyenne 20 déplacements et 159 kilomètres par personne et par semaine, soit environ 7990 kilomètres par an. $24 \%$ de cette distance correspond à des déplacements domicile-travail ou domicile-études. Dès lors, en première approche, la mobilité locale représente $99 \%$ des déplacements pour $59 \%$ des distances parcourues, et la mobilité sur longue distance $1 \%$ pour $41 \%$. Cette structure est 
restée identique entre les deux périodes. Les modes utilisés varient en conséquence (cf. tableau 1). La prépondérance de la voiture est nettement moins accusée sur la longue distance, cela au profit notamment de l'avion et du train.

Mobilité locale et mobilité sur longue distance s'avèrent donc très différentes selon leurs fréquences, leurs motifs et les modes utilisés. Les émissions de $\mathrm{CO}_{2}$ qu'elles génèrent ne s'expliqueront donc pas de la même manière. De même, les facteurs socioéconomiques individuels explicatifs de ces deux catégories de mobilité sont différents et deux typologies individuelles distinctes ont été construites pour en rendre compte.

\section{Deux typologies des individus en fonction de leurs mobilités locale et sur longue distance}

Un préalable est de rappeler les principaux acquis de la littérature dans ce domaine.

Les facteurs explicatifs le plus souvent mis en avant par cette abondante littérature (Orfeuil, 2000) sont les suivants :

Le statut (scolaire, étudiant, actif, chômeur, au foyer ou retraité), se recoupe avec l'âge et le cycle de vie, et conditionne les activités qui structurent et rythment la vie quotidienne (Orfeuil, 2002; Kaufman et Flamm, 2002). En matière de mobilité, ce facteur joue d'abord sur le nombre et les motifs de déplacements. Mais il affecte aussi les distances parcourues car les distances moyennes des déplacements peuvent être très différentes suivant les motifs. Les déplacements domicile-travail, notamment, sont beaucoup plus longs que les autres.

Du fait d'une répartition des tâches toujours marquée entre homme et femme au sein du ménage, le genre affecte également le nombre et les motifs des déplacements quotidiens des adultes (Hjorthol, 2003). Mais il joue aussi sur les distances parcourues : par exemple, le lieu d'emploi des femmes actives reste significativement plus proche du domicile que celui de leurs conjoints, même si cette tendance s'atténue progressivement au fil du temps (Bouzouina et al., 2011 ; Quételard, 2010).

Le niveau de revenu du ménage joue sur le niveau de mobilités, surtout s'il est mesuré en termes de distances parcourues. Cet effet richesse joue à plein pour la mobilité à longue distance, dans la mesure où un revenu élevé ouvre de larges possibilités en matière de mobilités de loisirs (Rouquette, 2001 ; Viard, 2002). Ce facteur concerne également, mais de manière beaucoup plus atténuée, la mobilité quotidienne : là aussi, les personnes les plus aisées, toutes autres

Tableau 1

Répartition des distances parcourues localement et sur longue distance selon le mode

\begin{tabular}{|c|c|c|c|}
\hline & 1994 & 2008 & $\begin{array}{c}\text { Évolution 94/08 } \\
\text { (en \%) }\end{array}$ \\
\hline \multicolumn{4}{|c|}{ Mobilité locale } \\
\hline Distance parcourue (mds voy.km/an) & 439 & 500 & 13,90 \\
\hline \multicolumn{4}{|l|}{ Dont (répartition en \%) : } \\
\hline Voiture Particulière & 86,00 & 85,50 & \\
\hline Transports Collectifs & 9,00 & 9,80 & \\
\hline Marche à pied & 2,70 & 2,80 & \\
\hline Autres modes & 2,30 & 2 & \\
\hline \multicolumn{4}{|c|}{ Mobilité sur longue distance } \\
\hline Distance parcourue (mds voy.km/an) & 278 & 331 & 19,06 \\
\hline \multicolumn{4}{|l|}{ Dont (répartition en \%) : } \\
\hline Voiture Particulière & 52,20 & 51,20 & \\
\hline Avion & 27,60 & 30,10 & \\
\hline Train & 11,90 & 14,10 & \\
\hline Autres modes & 8,30 & 4,60 & \\
\hline Mobilité totale (mds voy.km/an) & 717 & 828 & 15,48 \\
\hline
\end{tabular}

Lecture: en 1994, les mobilités locales ont représenté 439 milliards de voyageurs*kilomètres, dont $86 \%$ ont été réalisés en voiture

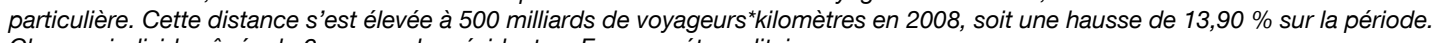
Champ : individus âgés de 6 ans ou plus résidant en France métropolitaine.

Source: traitements LET-Certu à partir de Insee-Inrets: enquête nationale Transports 1994, et de Insee-Inrets-SoeS (Service de l'Observation et des Statistiques du Ministère de l'Ecologie, du Développement Durable et de l'Energie) : enquête nationale Transports et déplacements 2008 . 
choses égales par ailleurs, tendent à parcourir des distances un peu plus longues, même si la forte proportion de déplacements contraints, en partie indépendants du niveau de revenu, limite les écarts - par exemple, les actifs modestes ne parcourent pas de distances quotidiennes plus faibles que les actifs aisés (Paulo, 2006 ; Nicolas et David, 2009).

La densité et la mixité fonctionnelle de la zone de résidence du ménage impactent les distances à parcourir (voir Fouchier, 1997, ou Pouyanne, 2004, pour l'effet de la densité ; Cervero et Kockelman, 1997, ou Rajamani et al., 2003 pour la mixité). C'est également le cas de la nature et de la performance des réseaux de transport. Ces facteurs jouent ainsi sur les modes utilisés (Cervero, 2002; Joly et al., 2009). Dans les espaces centraux, denses et bien équipés en transports collectifs, les distances parcourues sont plus courtes et les pratiques modales beaucoup plus diversifiées. Ces facteurs apparaissent beaucoup moins explicatifs des déplacements à longue distance. Ils joueraient même dans ce cas dans l'autre sens : on peut ainsi s'interroger sur la mobilité de week-end des habitants des centres urbains, qui pourraient avoir tendance à davantage rechercher des espaces éloignés que les périurbains, plus centrés sur leur résidence. Ainsi les écarts de distances parcourues quotidiennement en semaine et d'émissions correspondantes tendraient-ils à être compensés le week-end (Orfeuil et Soleyret, 2002).

L'accès à l'automobile apparaît comme une variable secondaire, qui dépend en amont du niveau de revenu et du lieu de localisation résidentielle (Dupuy, 1999). Cependant, c'est une variable déterminante car si l'usage de la voiture est bien généralisé aujourd'hui (Boucq et al., 2011), les personnes qui n'y ont pas accès parcourent des distances quotidiennes nettement plus faibles que les autres, et ce, même à caractéristiques socio économiques similaires. De même, l'automobile est un mode de transport important pour faciliter la mobilité à longue distance (Paulo, 2006).

Différents travaux menés au sein du Laboratoire d'Économie des Transports (par exemple, Nicolas et al., 2001 ; Paulo, 2006 ; ou Vanco, 2011, à partir des enquêtes locales; Mézière, 2004, Raux et al., 2005, 2006, ou Paulo, 2006, sur l'ENT de 1994) retrouvent ces principaux facteurs explicatifs des mobilités individuelles. Ils proposent des typologies des individus en fonction de leurs mobilités, et permettent ainsi de hiérarchiser le pouvoir explicatif des différents facteurs tout en prenant en compte leur poids relatif selon le groupe d'individus considéré. Ils se fondent sur des analyses de la variance des distances parcourues (Nicolas et al., 2001 ; Mézière, 2004 ; Raux et al., 2006). Nous avons retenu ainsi deux typologies faisant ressortir les facteurs socioéconomiques explicatifs des mobilités locale et à longue distance et destinées à servir de grilles de lecture aux résultats de nos analyses (cf. tableaux 2 et 3 ).

La définition de certaines des variables construites pour établir ces deux typologies est précisée ci-dessous.

Activité : être actif et occuper un emploi.

Motorisation: avoir le permis de conduire et appartenir à un ménage motorisé. Dans le cas d'un ménage motorisé dans lequel le nombre de titulaires du permis est supérieur à celui des véhicules, ne sont considérées comme motorisées que les personnes déclarant utiliser régulièrement une voiture comme moyen de locomotion.

Revenu: le revenu déclaré par chaque ménage est ramené à un revenu par unité de consommation à partir des équivalences établies par l'Insee ( 1 pour le premier adulte, 0,5 pour les autres individus de 14 ans ou plus, et 0,3 pour les individus de moins de 14 ans ; voir Hourriez et Ollier, 1997). Trois classes de revenu ont ensuite été distinguées en découpant les ménages en tiers aisé, médian et modeste.

Localisation: à la suite d'un précédent travail mené sur l'ENT de 1994 (Raux et al., 2006), trois classes de communes ont été construites en croisant distance au centre et densité de la commune de résidence (communes denses et centrales, zones périphériques peu ou moyennement denses, zones rurales ou périurbaines, très peu denses, cf. tableau 4).

\section{Les émissions de $\mathrm{CO}_{2}$ liées à la mobilité des personnes ont augmenté de $14 \%$ entre 1994 et 2008}

E 1 termes d'émissions de $\mathrm{CO}_{2}$, en 1994, sur et par an pour se déplacer, $1233 \mathrm{~kg}(69 \%)$ proviennent du local et $550 \mathrm{~kg}(31 \%)$ de la 
longue distance (cf. tableau 5). Sur la longue distance, les fortes émissions unitaires de l'avion (mesurées en $\mathrm{g} /$ pass.km) sont compensées par une émission moyenne de l'automobile plus faible qu'au plan local (notamment liée à un taux de remplissage plus élevé pour ce type de déplacement), et par la remarquable modération du train et de l'autocar en matière d'émission (cf. encadré 2).

Ces deux types de mobilité diffèrent également quant à l'évolution de leurs émissions entre 1994 et 2008 : la croissance de ces dernières est sensiblement plus réduite sur la longue distance (+ $8 \%$ ) qu'au plan local (+17\%) (cf. tableau 5). La croissance démographique $(+5,9 \%$ entre 1994 et 2008) est un premier facteur explicatif de cette hausse des émissions qui vaut pour les deux types de mobilité.

Ensuite, concernant la mobilité locale, la modération de la croissance des émissions par personne s'explique par la progression ellemême modérée des facteurs dont elle résulte (cf. tableau 1). Le nombre de déplacements par personne reste stable $(-1 \%)$ mais les distances, par contre, s'allongent $(+9 \%)$ : l'étalement urbain se poursuit, même si c'est de manière ralentie par rapport à la période précédente (Hubert et Delisle, 2010). Enfin, on constate une hausse des émissions unitaires moyennes (+3\%), imputable notamment à une diminution du taux d'occupation des automobiles insuffisamment contrebalancée par la faible baisse des consommations unitaires des véhicules (cf. infra).

A contrario, pour la mobilité à longue distance, les émissions unitaires décroissent de manière perceptible $(-10 \%)$, traduisant d'une part le poids grandissant du train et particulièrement du TGV et, d'autre part et surtout, les améliorations enregistrées au niveau de l'avion (meilleurs taux de remplissage et progrès technique).

Tableau 2

Typologie explicative des mobilités individuelles locales

\begin{tabular}{|c|c|c|c|}
\hline Groupe & $\begin{array}{l}\text { Population totale } \\
\text { (1994) }\end{array}$ & $\begin{array}{c}\text { Échantillon enquêté } \\
\text { (1994) }\end{array}$ & $\begin{array}{c}\text { Budget-distance } \\
(\mathrm{km} / \mathrm{j} \text { en 1994) }\end{array}$ \\
\hline Écolier du primaire, centres et périphérie & 2023431 & 299 & 11,0 \\
\hline périurbain & 1694232 & 289 & 15,9 \\
\hline Élève du secondaire, centres et périphérie & 4098140 & 581 & 12,4 \\
\hline périurbain & 2592118 & 378 & 21,6 \\
\hline Étudiant, centres & 1079657 & 208 & 20,2 \\
\hline périphéries et périurbain & 1001065 & 189 & 32,1 \\
\hline Actif(ve) non motorisé(e) & 3442948 & 811 & 17,2 \\
\hline Femme active, motorisée centres & 2015505 & 620 & 24,5 \\
\hline motorisée périphérie & 2338107 & 722 & 28,7 \\
\hline motorisée périurbain & 2628284 & 804 & 35,3 \\
\hline Homme actif, motorisé centres & 3135448 & 746 & 31,0 \\
\hline motorisé périphérie & 3388928 & 819 & 33,2 \\
\hline motorisé périurbain & 4600339 & 1144 & 43,3 \\
\hline Personne inactive, non motorisée & 3767285 & 593 & 10,3 \\
\hline motorisée centres et périphérie & 2252836 & 501 & 23,1 \\
\hline motorisée périurbain & 1614597 & 374 & 25,5 \\
\hline Retraité(e) non motorisé(e) & 4790683 & 841 & 6,1 \\
\hline motorisé(e), $<75$ ans centres & 966040 & 244 & 15,7 \\
\hline motorisé(e), $<75$ ans périphérie périurbain & 3329250 & 826 & 19,9 \\
\hline motorisé(e), 75 ans et plus & 550817 & 142 & 10,1 \\
\hline Autres & 1724202 & 305 & 14,8 \\
\hline Total & 53033913 & 11436 & 21,9 \\
\hline
\end{tabular}

Lecture : typologie en 21 groupes, constituée à partir des principaux facteurs explicatifs des mobilités locales (statut de la personne, localisation résidentielle, genre, accès au volant et âge pour les retraités).

Champ : individus âgés de 6 ans ou plus résidant en France métropolitaine.

Source : traitement LET-Certu à partir de Insee-Inrets : enquête nationale Transports 1994, et de Insee-Inrets-SoeS : enquête nationale Transports et déplacements 2008. 
La longueur moyenne des déplacements évolue peu $(+1,2 \%)$, et c'est la croissance de leur nombre $(+12 \%$ en moyenne par personne et par an) qui annihile ces progrès.

Ainsi, même si les distances parcourues ont crû plus fortement en longue distance qu'en local, c'est à ce dernier niveau que les émissions augmentent le plus sur la période $(+17 \%$ contre $+8 \%$ ).

\section{Les facteurs explicatifs des évolutions des émissions de $\mathrm{CO}_{2}$ liées à la mobilité locale}

T e croisement entre les différents groupes de la typologie présentée plus haut et les facteurs explicatifs des émissions permettent d'analyser à un niveau assez détaillé les

Tableau 3

Typologie explicative des mobilités individuelles à longue distance

\begin{tabular}{|c|c|c|c|}
\hline Groupe & $\begin{array}{l}\text { Population totale } \\
\text { (1994) }\end{array}$ & $\begin{array}{c}\text { Échantillon enquêté } \\
\text { (1994) }\end{array}$ & $\begin{array}{l}\text { Budget-distance } \\
\text { (km/an en 1994) }\end{array}$ \\
\hline Scolarisé aisé & 2730390 & 331 & 6706 \\
\hline Scolarisé médian & 3403845 & 299 & 3506 \\
\hline Scolarisé modeste & 4273685 & 256 & 3497 \\
\hline Étudiant & 2080722 & 294 & 7181 \\
\hline Actif aisé, centre et périphérie & 6520978 & 1658 & 11039 \\
\hline Actif aisé périurbain & 2551892 & 651 & 7264 \\
\hline Actif médian & 8106673 & 1280 & 4811 \\
\hline Actif modeste centre & 1156513 & 161 & 4415 \\
\hline Actif modeste périphérie & 1114061 & 145 & 3875 \\
\hline Actif modeste périurbain & 2099442 & 224 & 2618 \\
\hline Inactif aisé / médian centre & 1249053 & 180 & 9357 \\
\hline Inactif aisé / médian périphérie & 1228151 & 179 & 4574 \\
\hline Inactif aisé/ médian périurbain & 1297426 & 210 & 5318 \\
\hline Inactif modeste & 3860088 & 307 & 2080 \\
\hline Retraité $<75$ ans aisé & 2443816 & 529 & 7066 \\
\hline Retraité $<75$ ans médian & 2580732 & 340 & 3013 \\
\hline Retraité $<75$ ans modeste & 2363871 & 214 & 1670 \\
\hline Retraité 75 ans+ aisé & 530650 & 50 & 2044 \\
\hline Retraité 75 ans+ médian / modeste & 1717721 & 108 & 1118 \\
\hline Autre & 1724202 & 171 & 2422 \\
\hline Total & 53033913 & 7587 & 5147 \\
\hline
\end{tabular}

Lecture : typologie en 20 groupes, constituée à partir des principaux facteurs explicatifs des mobilités à longue distance (statut de la personne, niveau de revenu, localisation résidentielle et âge). Les termes " aisé ", "médian » et "modeste " désignent les quantiles d'ordre 1/3 (tiers) de revenu.

Champ : individus âgés de 6 ans ou plus résidant en France métropolitaine.

Source : traitement LET-Certu à partir de Insee-Inrets : enquête nationale Transports 1994, et de Insee-Inrets-SoeS : enquête nationale Transports et déplacements 2008.

Tableau 4

Classes de communes retenues pour construire les typologies

\begin{tabular}{|l|l|}
\hline \multicolumn{1}{|c|}{ Densité (hab/km2) } & \multicolumn{1}{|c|}{$0-10$} \\
\hline $0-100$ & Distance à la commune centre $(\mathrm{km})$ \\
$100-500$ & zural et périurbain \\
$500-3000$ & zones \\
3000 et plus & périphériques \\
\hline
\end{tabular}

Source : à partir de Raux et al. (2006). 
évolutions des émissions de $\mathrm{CO}_{2}$ liées à la mobilité locale $(+17 \%$ entre 1994 et 2008).

Dans la périphérie des villes, la voiture est le principal responsable de la hausse des émissions, alors que dans les espaces centraux leur recul s'explique par une diversification des modes

Les émissions progressent de $11 \%$ au niveau individuel, et de $17 \%$ au niveau global du fait d'une croissance de $6 \%$ de la population sur la période (cf. tableau 6). C'est l'allongement des distances qui tire le plus les émissions vers le haut $(+8 \%)$, tandis que l'augmentation reste plus limitée tant pour le nombre de déplacements par personne et que pour les émissions unitaires (respectivement $+1 \%$ et $+3 \%$ ). Ceci s'explique par une augmentation de la population plus rapide dans les zones périurbaines et dans les zones périphériques que dans les espaces centraux (la part relative de ces différentes zones dans l'ensemble de la population a respectivement augmenté de $+9,6,+4,1$ et $+1,9 \%$ ). S'ajoute à cela le fait que les distances parcourues (par jour et par personne) ont, elles aussi, plus augmenté dans le périurbain et le périphérique que dans le centre $(+8,2 \%$, $+12,8$ et $+1,1 \%)$.

Sous l'angle du mode de transport, si l'on exclut la catégorie marginale des «autres modes » $(2 \%$ des distances et $1 \%$ des émissions), l'automobile se conjugue à la poursuite de la périurbanisation pour tirer les émissions vers le haut. En matière de mobilité locale, l'accentuation de la dépendance automobile s'est poursuivie entre 1994 et 2008 , ce qui ressort à la fois du nombre de déplacements individuels et des distances parcourues par déplacement (cf. tableau 6). Les émissions unitaires par passager.kilomètre ont également continué à croître, ce qui traduit la baisse du taux de remplissage et l'usage toujours plus individualisé de la voiture qui a compensé la diminution des émissions unitaires des véhicules. L'usage

Tableau 5

Évolution des émissions individuelles de $\mathrm{CO}_{2}$ entre 1994 et 2008

\begin{tabular}{|l|c|c|c|c|c|c|}
\hline & \multicolumn{3}{|c|}{ Mobilité locale } & \multicolumn{3}{c|}{ Mobilité sur la longue distance } \\
\cline { 2 - 7 } & 1994 & 2008 & $\begin{array}{c}\text { Évolution } \\
1994-2008 \\
(\%)\end{array}$ & 1994 & 2008 & $\begin{array}{c}\text { Évolution } \\
1994-2008 \\
(\%)\end{array}$ \\
\hline Nombre de déplacements (pers. /an) & 1024 & 1012 & -1 & 12,6 & 14,1 & 12 \\
Distance moyenne par déplacement (km) & 8,1 & 8,78 & 8 & 414 & 419 & 1 \\
Émissions unitaire $\mathrm{CO}_{2}$ (g/pass.km) & 149 & 154 & 3 & 105 & 95 & -10 \\
Émissions $\mathrm{CO}_{2}$ (kg/an/pers.) & 1233 & 1366 & 11 & 550 & 560 & 2 \\
Population totale (millions) & 53 & 56 & 6 & \multicolumn{2}{|c|}{ idem } & \\
Émissions totales (millions de tonnes) & 65 & 77 & 17 & 29 & 31 & 8 \\
\hline
\end{tabular}

Champ : individus âgés de 6 ans ou plus résidant en France métropolitaine.

Source : traitement LET-Certu à partir de Insee-Inrets : enquête nationale Transports 1994, et de Insee-Inrets-SoeS : enquête nationale Transports et déplacements 2008.

Tableau 6

Coefficients d'évolution des émissions de $\mathrm{CO}_{2}$ liées à la mobilité locale par modes (ENT 1994 et 2008)

\begin{tabular}{|c|c|c|c|c|c|c|}
\hline & $\begin{array}{c}\text { Émissions } \\
\mathrm{kg} / \mathrm{j} / \text { pers } 94\end{array}$ & $\begin{array}{l}\text { Évolution } \\
\text { nbdep/pers }\end{array}$ & $\begin{array}{l}\text { Évolution } \\
\text { dist/dep }\end{array}$ & $\begin{array}{c}\text { Évolution émissions } \\
\text { unitaires }\end{array}$ & $\begin{array}{l}\text { Évolution } \\
\text { émis/pers/j }\end{array}$ & $\begin{array}{l}\text { Émissions } \\
\mathrm{kg} / \mathrm{j} / \text { pers } 08\end{array}$ \\
\hline Voiture particulière & 3,23 & 0,99 & 1,07 & 1,05 & 1,11 & 3,6 \\
\hline Transports collectifs urbains & 0,12 & 1,01 & 1,16 & 0,71 & 0,83 & 0,1 \\
\hline Modes doux & - & 0,96 & 1,16 & - & & - \\
\hline Autres modes & 0,03 & 0,93 & 0,95 & 1,38 & 1,33 & 0,04 \\
\hline Tous modes & 3,38 & 0,99 & 1,08 & 1,03 & 1,11 & 3,74 \\
\hline
\end{tabular}

Champ : individus âgés de six ans ou plus résidant en France métropolitaine.

Source : traitement LET-Certu à partir de Insee-Inrets : enquête nationale Transports 1994, et de Insee-Inrets-SoeS : enquête nationale Transports et déplacements 2008. 
des transports collectifs urbains reste stable au niveau individuel : les personnes motorisées tendent à les délaisser encore un peu plus, quelle que soit leur zone de résidence, tandis que celles qui n'utilisent pas de voiture y ont davantage recours qu'auparavant. Surtout, les élèves du secondaire et les étudiants, principaux usagers de ce mode, font preuve, en la matière, d'une permanence de comportement contribuant à stabiliser les évolutions globales. Les distances par déplacement augmentent plus nettement $(+16 \%)$ et sont tirées essentiellement par les résidents des zones centrales et périphériques. La croissance des transports collectifs urbains apparaît in fine plus importante que celle de l'automobile $(+24 \% v s$ $+13 \%$ en passagers.kilomètres). La première concerne essentiellement les zones centrales, la seconde, les espaces périphériques. Par contre, les différences en valeurs absolues jouent nettement en faveur de l'automobile (+ 9,5 mds pass.km vs +49 mds pass.km). En termes d'émissions de $\mathrm{CO}_{2}$, c'est bien sur la voiture que les enjeux restent centrés. En ce qui concerne les modes doux, enfin, les déplacements en marche à pied et à vélo accusent un léger repli. Les parts de ces modes de transport dans le nombre total de déplacements passent respectivement de $23,2 \%$ et $2,9 \%$ en 1994 à $22,3 \%$ et $2,7 \%$ en 2008 . Ces données globales ne doivent pas masquer leur regain dans les parties centrales des agglomérations qui se traduit également par la croissance des distances moyennes des déplacements qui leur sont liés $(+16 \%)$.

\section{L'augmentation du nombre et de la mobilité des femmes actives...}

On examine maintenant l'évolution des émissions et de leurs variables explicatives suivant les groupes de population homogènes sous l'angle des mobilités individuelles locales mis en évidence par la typologie retenue dans cette étude (cf. infra et tableau 2). Le fait d'être actif et d'être soumis à une mobilité contrainte dans l'espace et dans le temps s'avère un facteur explicatif décisif des émissions et de leurs évolutions. Les distances à parcourir pour réaliser les déplacements domicile travail sont, en effet, plus longs que la moyenne. La voiture s'impose alors comme une nécessité et sa diffusion s'est renforcée : $90 \%$ des actifs sont motorisés en 2008 contre $84 \%$ en 1994. Alors qu'ils représentent $45 \%$ de la population en 2008 , leurs émissions de $\mathrm{CO}_{2}$ correspondent à $65 \% \mathrm{du}$ total cumulé.
La dynamique des actifs et de leurs émissions est fortement déterminée par l'évolution du groupe des femmes actives (cf. tableau 7 et graphique I). Le groupe des hommes actifs progresse modérément du point de vue démographique $(+10 \%)$ et voit baisser ses émissions individuelles (- $5 \%$ d'émissions par personne). Par contre, le nombre de femmes actives a très fortement augmenté $(+50 \%)$ et leur comportement tend progressivement à rejoindre celui de leurs homologues masculins ( $+5 \%$ d'émissions par personne). Ainsi, d'une part, le taux d'activité des femmes a continué à se renforcer durant la période, ce qui se traduit également par une baisse sensible du groupe des personnes inactives (- $30 \%)$. D'autre part, la mobilité des femmes vivant en périphérie a continué à se tourner vers l'automobile, avec un nombre de déplacements stable, un allongement des distances et une utilisation de plus en plus individuelle de la voiture. Cette tendance est partiellement compensée par les pratiques des femmes actives résidant dans les zones centrales. Leur nombre de déplacements quotidiens a baissé fortement (- $31 \%$ ), comme pour l'ensemble des résidants centraux. Comme les distances qu'elles ont parcourues et leurs émissions unitaires sont restées stables, ces évolutions ont conduit à une baisse de $30 \%$ de leurs émissions locales.

In fine, la progression des émissions totales imputables aux actifs est alignée sur la moyenne générale et la stabilité de leur part dans les émissions totales $(66 \%$ en 1994 et $67 \%$ en 2008), recouvrent deux évolutions contraires - d'une part, la forte progression imprimée par la croissance du nombre de femmes actives et, d'autre part, la diminution des émissions des actifs centraux.

\section{... se conjugue à la diffusion générale de la voiture chez les retraités pour expliquer l'accroissement des émissions}

Le second groupe à connaître des évolutions marquées correspond à celui des retraités, dont les effectifs progressent nettement $(+28 \%)$, et dont la motorisation se renforce avec le renouvellement des générations $(50 \%$ des retraités avaient une voiture en 1994, $74 \%$ en 2008). La combinaison de ces deux facteurs, qui jouent chacun fortement, conduit à une contribution aux émissions en très nette progression entre les deux périodes : elle est passée de $10 \%$ en 1994 à $17 \%$ en 2008 . 
Ces évolutions sont sous-tendues par une importante utilisation de la voiture. Ainsi, même le nombre moyen de déplacements automobile double au sein du groupe des retraités qui n'y ont pas directement accès, avec une longueur qui s'accroît de $20 \%$. L'effet localisation joue légèrement sans parvenir à compenser ce mouvement et les retraités motorisés de moins de 75 ans connaissent une augmentation de leurs émissions moyennes d'environ $20 \%$ lorsqu'ils résident dans le centre des zones urbaines et de $30 \%$ lorsqu'ils résident dans les zones moins denses. Enfin, si les émissions des personnes motorisées de 75 ans et plus restent quantitativement relativement stables (+6\%), leur nombre augmente de manière extrêmement forte $(+330 \%)$, ce qui témoigne autant de l'irruption massive de la voiture dans l'existence des personnes relevant de cette classe d'âge que de l'allongement de l'espérance de vie.

\section{La stabilité apparente des émissions imputables aux inactifs, aux étudiants et aux élèves du primaire et du secondaire recouvre souvent des contrastes entre sous-catégories}

En ce qui concerne le groupe des inactifs, l'évolution la plus marquante réside dans la baisse de ses effectifs $(-30 \%)$, qui renvoie à la poursuite de la hausse du taux d'activité féminin évoquée précédemment. Ce groupe se signale aussi par des évolutions contrastées entre les espaces centraux et les zones moins denses. Les personnes inactives des centres ont vu leurs émissions moyennes baisser de $41 \%$ : cela s'explique notamment par une forte baisse du nombre de déplacements par personne (- $43 \%)$, conjuguée à une diminution des distances parcourues $(-9 \%)$ et des émissions unitaires, notamment automobile (-42\%). Dans l'autre sens, les inactifs des zones moins denses connaissent

Tableau 7

Coefficients d'évolution des émissions de $\mathrm{CO}_{2}$ liées à la mobilité locale par groupes de population (ENT 1994 et 2008)

\begin{tabular}{|c|c|c|c|c|c|c|c|}
\hline & \multirow[b]{2}{*}{$\begin{array}{c}\text { Population } \\
1994\end{array}$} & \multirow[b]{2}{*}{$\begin{array}{l}\text { Émissions } \\
\text { (kg/pers/j) }\end{array}$} & \multicolumn{5}{|c|}{ Coefficients d'évolution entre 1994 et 2008} \\
\hline & & & Pop. & $\begin{array}{l}\mathrm{Nb} \text { deplt / } \\
\text { pers }\end{array}$ & $\begin{array}{l}\text { Dist/ } \\
\text { deplt }\end{array}$ & $\begin{array}{c}\text { Émissions } \\
\text { unitaire }\end{array}$ & $\begin{array}{c}\text { Émissions } \\
\text { totales }\end{array}$ \\
\hline Primaire centre périphérie & 2023431 & 0,9 & 1,07 & 0,76 & 1,04 & 1,06 & 0,91 \\
\hline Primaire périurbain & 1694232 & 1,2 & 0,96 & 0,84 & 1,35 & 1,11 & 1,21 \\
\hline Secondaire centre périphérie & 4098140 & 1,1 & 0,81 & 1,18 & 1,22 & 0,81 & 0,95 \\
\hline Secondaire périurbain & 2592118 & 1,6 & 0,91 & 1,09 & 0,99 & 1,08 & 1,06 \\
\hline Étudiant centre & 1079657 & 2,5 & 1,22 & 0,87 & 0,91 & 0,80 & 0,77 \\
\hline Étudiant périphérie et périurbain & 1001065 & 4,2 & 1,28 & 0,94 & 1,06 & 0,93 & 1,18 \\
\hline Actif(ve) non motorisé(e) & 3442948 & 1,4 & 0,74 & 1,86 & 0,99 & 0,83 & 1,14 \\
\hline Femme active, motorisée centre & 2015505 & 4,3 & 1,48 & 0,69 & 1,02 & 1,01 & 1,04 \\
\hline Femme active motorisée périphérie & 2338107 & 4,9 & 1,33 & 1,05 & 1,07 & 1,07 & 1,60 \\
\hline Femme active motorisée périurbain & 2628284 & 5,4 & 1,66 & 1,00 & 1,11 & 1,02 & 1,87 \\
\hline Homme actif, motorisé centre & 3135448 & 5,8 & 1,13 & 0,80 & 0,98 & 0,99 & 0,86 \\
\hline Homme motorisé périphérie & 3388928 & 5,8 & 1,06 & 0,92 & 1,25 & 1,01 & 1,22 \\
\hline Homme motorisé périurbain & 4600339 & 6,6 & 1,11 & 0,89 & 0,99 & 1,07 & 1,04 \\
\hline Personne inactive non motorisée & 3767285 & 0,9 & 0,50 & 1,29 & 0,95 & 0,85 & 0,52 \\
\hline Personne inactive motorisée centre et périphérie & 2252836 & 3,6 & 0,94 & 0,57 & 0,91 & 1,13 & 0,55 \\
\hline Personne inactive motorisée périurbain & 1614597 & 3,5 & 0,82 & 0,78 & 1,35 & 1,03 & 0,89 \\
\hline Retraité(e) non motorisé(e) & 4790683 & 0,5 & 0,71 & 1,90 & 1,16 & 1,03 & 1,62 \\
\hline Retraité(e) motorisé(e), <75 ans centre & 966040 & 2,6 & 1,49 & 0,95 & 1,21 & 1,04 & 1,79 \\
\hline Retraité(e) motorisé(e), <75 ans périphérie & 3329250 & 3,1 & 1,54 & 1,16 & 1,08 & 1,04 & 2,00 \\
\hline Retraité(e) motorisé(e), 75 ans et + & 550817 & 1,5 & 4,30 & 0,72 & 1,26 & 1,18 & 4,58 \\
\hline Autres (personne handicapée,...) & 1724202 & 1,7 & 0,69 & 0,97 & 0,85 & 1,23 & 0,71 \\
\hline Moyenne & 53033913 & 3,1 & 1,06 & 1,02 & 1,09 & 1,03 & 1,21 \\
\hline
\end{tabular}

Champ : déplacements des individus âgés de 6 ans ou plus résidant en France métropolitaine; émissions calculées avec le taux de remplissage déclaré, enfants de moins de 6 ans inclus.

Source : traitement LET-Certu à partir de Insee-Inrets : enquête nationale Transports 1994, et de Insee-Inrets-SoeS : enquête nationale Transports et déplacements 2008. 
une hausse de leurs émissions moyennes $(+9 \%)$. Leur nombre de déplacement moyen a également baissé mais dans de moindres proportions $(-22 \%)$; ils se sont allongés $(+35 \%)$ et

\section{Graphique I \\ Poids des modes dans l'évolution des émissions individuelles de $\mathrm{CO}_{2}$ liées à la mobilité locale, en $\mathrm{kg} /$ personne/jour par groupes de population (ENT 1994 et 2008)}

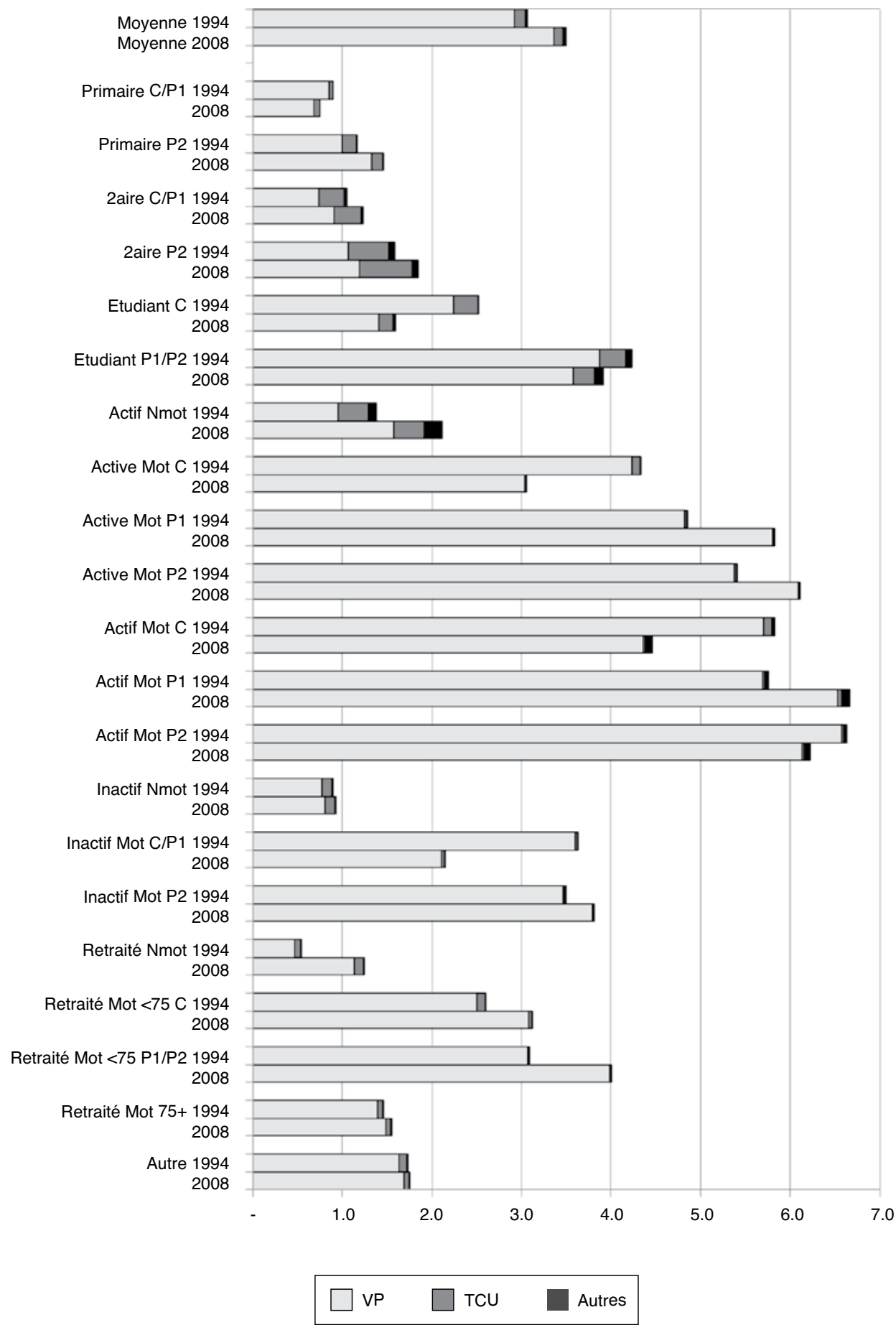

Lecture : $C$ : zones centrales ; $P 1$ : zones périphériques ; $P 2$ : zones périurbaines

En 1994, les écoliers du primaire vivant dans les zones centrales et périphériques ont une mobilité locale qui génère en moyenne 0,9 kg de $C O$ par personne et par jour, avec une prédominance extrêmement forte de l'automobile dans le bilan; cette moyenne est tombée à $0,75 \mathrm{~kg}$ en 2008 .

Champ : individus âgés de 6 ans ou plus résidant en France métropolitaine ; émissions calculées avec le taux de remplissage déclaré, enfants de moins de 6 ans inclus.

Source : traitement LET-Certu à partir de Insee-Inrets : enquête nationale Transports 1994, et de Insee-Inrets-SoeS : enquête nationale Transports et déplacements 2008. 
leurs émissions unitaires ont augmenté $(+3 \%)$, ce qui traduit là encore une utilisation de la voiture plus individuelle que par le passé.

Au total, les émissions moyennes des personnes inactives baissent légèrement $(-6 \%)$. Par contre, le recul des effectifs du groupe fait que sa contribution globale aux émissions de $\mathrm{CO}_{2}$ des déplacements locaux diminue fortement, passant de $11 \%$ en 1994 à $6 \%$ en 2008.

L'allongement de la durée des études explique l'augmentation sensible des effectifs du groupe des étudiants $(+25 \%)$. Les pratiques de mobilité des étudiants résidant dans les zones centrales denses se sont fortement restructurées autour des modes alternatifs à la voiture, avec une augmentation des distances parcourues par personne de $17 \%$ en transports collectifs urbains et de $54 \%$ en modes doux contre une baisse de $38 \%$ de ce même indicateur appliqué à l'automobile. En conséquence, les émissions par personne ont baissé de $37 \%$ au sein de ce sous-groupe. Les étudiants résidant dans les zones moins denses sont par contre restés beaucoup plus dépendants de la voiture et leurs émissions par tête n'ont baissé que de $8 \%$ au cours de la période. Ainsi, la baisse des émissions globales des étudiants du centre (- $23 \%)$ est compensée par la hausse de ceux de périphérie $(+18 \%)$, si bien que les émissions du groupe dans son ensemble restent stables $(+2 \%)$. Elles représentent environ $4 \%$ des émissions totales en 1994 et en 2008).

Enfin, la palme en matière de stabilité revient aux émissions des élèves de primaire et du secondaire. Cette stabilité se vérifie tant en niveau qu'en structure. En ce qui concerne des élèves du primaire, la distinction entre les zones de résidence permet là encore de percevoir une tendance à l'abandon de la voiture dans les zones plus denses des centres et de la proche périphérie des villes, ce qui n'est pas le cas dans le périurbain. Ceci se traduit par une baisse des émissions par personne de $15 \%$ dans les centres et par une hausse de $25 \%$ en périphérie. Les émissions globales de cette catégorie progressent de $6 \%$, comme la moyenne de la population, et restent à $2 \%$ du volume total.

Cette tendance ne se vérifie pas pour les élèves $\mathrm{du}$ secondaire. Dans leur cas, l'accompagnement en voiture a continué à se renforcer, quelle que soit la zone de résidence, entraînant une hausse de $18 \%$ de leurs émissions moyennes. Par contre, comme leur nombre baisse, les émissions globales du groupe restent constantes et leur part dans le volume total diminue légèrement, passant de 5,2 à $4,3 \%$.

\section{Les facteurs explicatifs des évolutions des émissions de $\mathrm{CO}_{2}$ liées à la mobilité sur longue distance}

$\mathbf{L}$ a mobilité sur longue distance recouvre des comportements et répond à des mécanismes différents. Les émissions globales imputables à ce type de déplacement progressent beaucoup moins que dans le cadre de la mobilité locale $(+8 \%)$. Certains des facteurs utilisés pour construire la typologie explicative de cette mobilité vont s'avérer particulièrement explicatifs des mécanismes et les évolutions des émissions : tel est le cas du revenu, de la zone de résidence et du croisement entre statut et cycle de vie. On constatera également de grandes tendances propres à chacun des modes et transversales aux groupes de la typologie, comme par exemple la croissance généralisée de l'usage de l'avion.

\section{Une mobilité longue distance en hausse, dont les effets sont compensés par des émissions unitaires en baisse}

Mesurée en nombre de déplacements par an et par personne, la mobilité à longue distance a augmenté de façon notable en passant de 12,6 à $14,1(+11 \%)$, et ce, au sein de presque toute la population (cf. tableau 8 ci-dessous). La portée des déplacements est restée quant à elle beaucoup plus stable $(-2 \%)$ : on ne va donc pas plus loin qu'avant, mais on y va plus souvent. Les tendances sont cependant beaucoup plus contrastées que pour la mobilité sur courte distance, ce qui ne sera pas sans effet sur les analyses par groupe. La croissance du nombre de déplacements est compensée par une diminution généralisée des émissions unitaires $(-9 \%)$ (cf. tableau 8).

L'automobile reste le mode majoritaire en termes de distances parcourues dans ce cadre de la mobilité sur longue distance $(52 \%$ puis $51 \%$ des passagers.kilomètres en 1994 et en 2008). Elle est le seul mode qui n'a pas connu d'évolution forte en matière d'émissions unitaires car les progrès du parc en matière de performance énergétique ont été compensés par la baisse des taux d'occupation (diminuant de 1,78 
à 1,61 personnes par véhicule, soit -11\%). En conséquence, sa part dans les émissions de $\mathrm{CO}_{2}$ a augmenté, passant de $49 \%$ à $55 \%$.

Le train est un mode extrêmement peu émetteur du fait de son recours à la traction électrique. Comme ce type de traction a encore été étendu durant la période, ses émissions unitaires ont continué à baisser (-11\%). Par ailleurs, le trafic a augmenté sensiblement, avec une progression de $21 \%$ du nombre de déplacement par personne et de $10 \%$ de leur portée. La part du train dans le trafic total reste limitée $(11,9 \%$ en $1994,14,1 \%$ en 2008), mais cette progression a malgré tout contribué à la baisse des émissions unitaires globales.

En 1994, l'avion constituait le plus gros émetteur de $\mathrm{CO}_{2}$ pour la mobilité à longue distance, avec la moitié des émissions totales. La forte augmentation de ce type de mobilité sur la période s'est faite en sa faveur, avec une croissance de $+26 \%$ du nombre de déplacements en avion par personne entre 1994 et 2008. En termes d'émissions de $\mathrm{CO}_{2}$, cette progression a cependant été compensée par une légère baisse des distances par déplacement (- $2 \%)$ et, surtout, par une forte baisse des émissions unitaires (- $25 \%)$ s'expliquant à la fois par les progrès techniques en matière de performance énergétique et par de plus forts taux de remplissage. Ces progrès sont cohérents avec l'estimation d'une baisse de $32 \%$ des émissions par voyageur.kilomètre entre 1990 et 2009 établie par la DGAC et le CITEPA (DGAC, 2010 ; Chèze et al., 2011), même si c'est sans doute à ce niveau que les incertitudes liées à nos hypothèses de calcul sont les plus fortes : d'une part la mesure des distances par du vol d'oiseau ne prend pas en compte l'éventualité d'une augmentation de la proportion de voyages passant par des hubs; d'autre part les bases de données d'émissions utilisées sont moins homogènes dans le temps que pour l'automobile, ce qui peut également induire des variations selon les méthodes utilisées. Ces variations peuvent avoir des répercussions importantes puisque, compte tenu du poids de l'avion dans les émissions liées à la mobilité sur longue distance, une variation de $\pm 10 \%$ des hypothèses d'évolution des émissions unitaires ou des distances amène soit à une stabilité soit à une augmentation de $+10 \%$ des émissions totales de cette catégorie de déplacement. Les conclusions qui peuvent être tirées de l'examen des mobilités sur la longue distance doivent donc être maniées avec précaution.

\section{Une baisse généralisée des émissions dans tous les groupes, sauf pour les retraités qui renforcent leur usage de la voiture}

La croissance des émissions de $\mathrm{CO}_{2}$ imputables à la mobilité à longue distance est principalement tirée par les personnes retraitées. En effet, tous les sous-groupes de cette catégorie voient leurs émissions augmenter, au contraire du reste de la population. Le même constat prévaut qu'au plan local : le renouvellement des générations a conduit à la diffusion de la voiture dans cette classe d'âge et le nombre de déplacements automobile a fortement progressé $(+64 \%)$, avec des portées et des émissions qui restent stables (respectivement $-2,5 \%$ et $+1 \%$ ). Combinées avec la croissance démographique des seniors, ces dynamiques conduisent dès lors à une multiplication par deux des émissions du groupe (cf. tableau 9 et graphique II).

Parallèlement à cette tendance générale, des inflexions particulières peuvent être mises en évidence suivant le niveau de revenu et l'âge. Les moins de 75 ans aisés, de loin les plus mobiles du groupe, utilisaient déjà régulièrement

Tableau 8

Coefficients d'évolution des émissions de $\mathrm{CO}_{2}$ liées à la mobilité à longue distance par modes (ENT 1994 et 2008)

\begin{tabular}{|l|c|c|c|c|c|c|c|}
\hline & $\begin{array}{c}\text { Émissions } \\
\mathrm{kg} / \mathrm{an} / \text { pers 94 }\end{array}$ & $\begin{array}{c}\text { Évolution } \\
\text { nbdep/pers }\end{array}$ & $\begin{array}{c}\text { Évolution } \\
\text { dist/dep }\end{array}$ & $\begin{array}{c}\text { Évolution } \\
\text { émis. unitaire }\end{array}$ & $\begin{array}{c}\text { Émissions } \\
\mathrm{kg} / \mathrm{an} / \mathrm{pers} \text { 08 }\end{array}$ & $\begin{array}{c}\text { Évolution } \\
\text { émis/pers/an }\end{array}$ & $\begin{array}{c}\text { Évolution } \\
\text { totale }\end{array}$ \\
\hline Voiture particulière & 269 & 1,13 & 0,98 & 1,05 & 311 & 1,16 & 1,22 \\
Train & 7 & 1,21 & 1,10 & 0,89 & 8 & 1,20 & 1,26 \\
Avion & 244 & 1,26 & 0,98 & 0,75 & 226 & 0,93 & 0,98 \\
Autres modes & 29 & 0,75 & 0,83 & 0,80 & 15 & 0,50 & 0,53 \\
\hline Tous modes & 550 & 1,11 & 1,01 & 0,91 & 560 & 1,02 & 1,08 \\
\hline
\end{tabular}

Champ : individus âgés de 6 ans ou plus résidant en France métropolitaine.

Source : traitement LET-Certu à partir de Insee-Inrets : enquête nationale Transports 1994, et de Insee-Inrets-SoeS : enquête nationale Transports et déplacements 2008. 
l'avion en 1994 (0,72 déplacement par an par personne contre 0,16 pour tous les autres groupes réunis). Leur prédilection pour ce mode a continué à s'intensifier (+ $76 \%)$, tandis que son usage ralliait d'autres groupes de retraités, avec une progression encore plus forte $(+109 \%$ pour les moins de 75 ans de revenus médians, $+122 \%$ pour les plus âgés aisés). Par contre, on constate une défection des plus modestes vis-à-vis de l'avion, quel que soit leur âge : - $20 \%$ chez ceux de moins de 75 ans (avec, en compensation, une augmentation de $50 \%$ leurs déplacements ferrés), mais surtout - $82 \%$ pour les plus de 75 ans à revenu médian ou modeste. Ainsi, les écarts de distances et de modes utilisés ne se résorbent que très partiellement entre les groupes, et ils s'accroissent même entre les plus aisés et les plus modestes.

Les actifs pèsent eux aussi lourdement dans les analyses globales puisqu'ils représentent $41 \%$ de la population et $58 \%$ des émissions de $\mathrm{CO}_{2}$ à longue distance en 1994 (respectivement $45 \%$ et $60 \%$ en 2008). Les émissions moyennes par personne ont baissé (- $7 \%$ ), mais le groupe a sensiblement augmenté $(+17 \%)$ et ses émissions totales sont donc en hausse $(+8 \%)$. Le croisement revenu / localisation introduit des distinctions significatives au sein de ce groupe, avec des écarts importants entre les actifs aisés des zones centrales et ceux des périphéries des zones urbaines, qui font 23 déplacements de plus de 80 kilomètres par an (dont 2 en avion) en 1994, émettant annuellement $1280 \mathrm{~kg}$ de $\mathrm{CO}_{2}$ par personne, et les actifs modestes des zones peu denses qui n'en réalisent que 9 ( 0,1 en avion) pour $230 \mathrm{~kg} \mathrm{de} \mathrm{CO}_{2}$. A partir de là, trois grandes tendances se dessinent :

D'un côté, la mobilité des plus aisés des zones denses n'augmente que de manière limitée ( $+6 \%$ de distance parcourue par personne), et

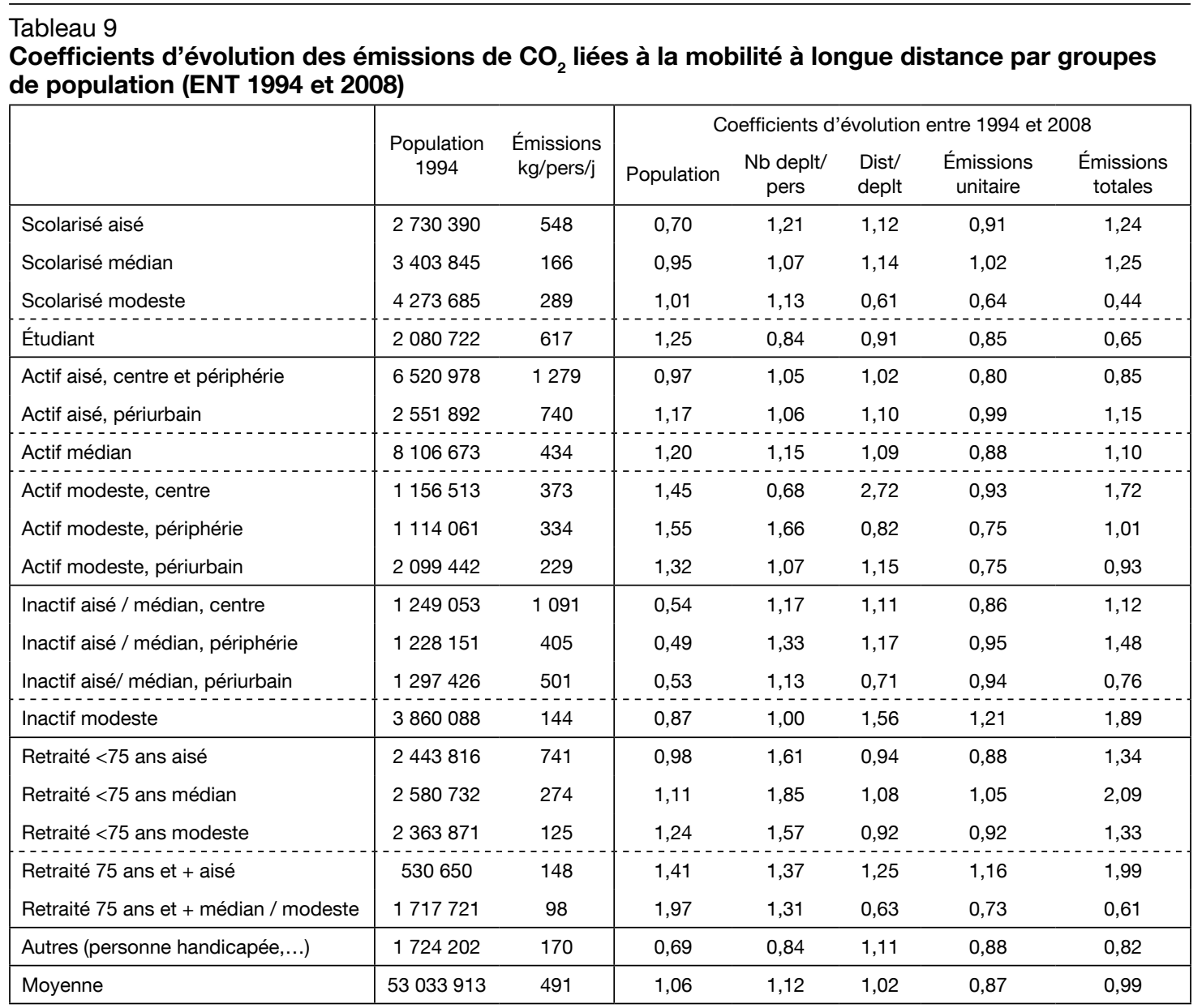

Lecture : " aisé », " médian » et " modeste » désignent les quantiles d'ordre 1/3 (tiers) de revenu, cf. dans le texte la description des deux typologies de ménages retenue pour cette étude.

Champ : individus âgés de 6 ans ou plus résidant en France métropolitaine; émissions calculées avec le taux de remplissage déclaré, enfants de moins de 6 ans inclus.

Source : traitement LET-Certu à partir de Insee-Inrets : enquête nationale Transports 1994, et de Insee-Inrets-SoeS : enquête nationale Transports et déplacements 2008. 
comme l'essentiel de leurs déplacements se fait en avion ( $42 \%$ des distances), moyen de transport dont les émissions ont fortement baissé, leurs émissions décroissent (- $15 \%)$.
Les actifs aisés du périurbains, ceux de revenus médians ou modestes résidant dans les zones centrales sont concernés par de fortes progressions : tout d'abord en distance parcourue par

\section{Graphique II}

Poids des modes dans l'évolution des émissions individuelles de $\mathrm{CO}_{2}$ liées à la mobilité à longue distance, en kg/personne/an, par groupes de population (ENT 1994 et 2008)

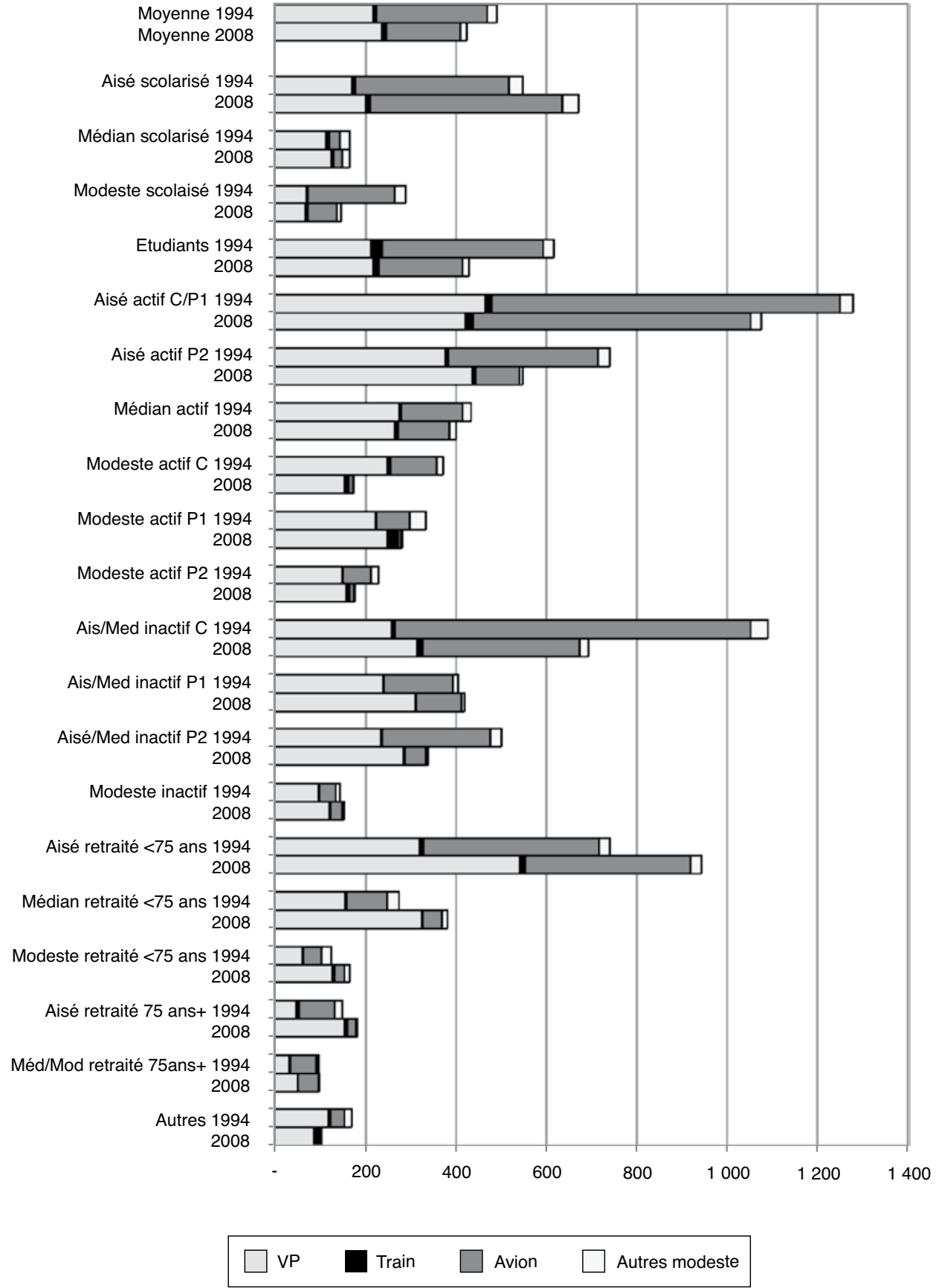

Lecture : $C$ : zones centrales ; $P 1$ : zones périphériques ; $P 2$ : zones périurbaines.

En 1994, les enfants scolarisés des ménages aisés ont une mobilité à longue distance qui génère en moyenne $550 \mathrm{~kg}$ de CO par personne et par an, dont $33 \%$ liés à leurs déplacements en automobile et $63 \%$ liés à leurs déplacements en avion; cette moyenne s'est élevée à $665 \mathrm{~kg}$ en 2008

Champ : individus âgés de 6 ans ou plus résidant en France métropolitaine ; émissions calculées avec le taux de remplissage déclaré, enfants de moins de 6 ans inclus.

Source : traitement LET-Certu à partir de Insee-Inrets : enquête nationale Transports 1994, et de Insee-Inrets-SoeS : enquête nationale Transports et déplacements 2008. 
personne et par an $(+28 \%)$ et ensuite en émissions, même si c'est de manière plus amortie $(+16 \%)$ du fait là encore de la baisse des émissions par personne de l'avion.

Enfin, dans les zones moins denses, les distances parcourues augmentent dans les mêmes proportions pour les personnes de revenu modeste que pour les autres $(+31 \%)$. Mais cette progression est essentiellement portée par le train dont l'usage, très limité en 1994, est multiplié par plus de $6^{1}$. Ce mode étant très peu émetteur, les émissions du groupe restent stables (- $2 \%)$.

Les poids des autres catégories dans les émissions de $\mathrm{CO}_{2}$ sont plus modestes, et leurs évolutions ont donc moins d'impact.

Ainsi, les inactifs ne représentent que $11 \%$ des émissions pour $14 \%$ de la population en 1994 (respectivement $9 \%$ et $9 \%$ en 2008). Dans ce groupe, une première distinction mérite d'être faite entre les inactifs aisés et médians d'une part et ceux à revenu modeste d'autre part. En effet, l'augmentation du taux d'activité féminin mentionné plus haut dans le cas de la mobilité locale se vérifie, en matière de mobilité sur la longue distance, essentiellement sur le segment des inactifs percevant les revenus les plus élevés: leurs effectifs diminuent de $48 \%$ en nombre, alors que cette baisse est beaucoup plus limitée chez les inactifs modestes (-13\%). Par ailleurs, les comportements de mobilité sur la longue distance sont bien différenciés entre les deux sous-groupes avec, en 1994, 6412 kilomètre par an pour les premiers contre 2080 pour les seconds. Ces écarts se résorbent partiellement sur la période, avec une croissance de $23 \%$ contre $56 \%$ des distances, et $11 \%$ contre $89 \%$ pour les émissions (la progression de l'avion dans les deux groupes limite la hausse chez les premiers qui l'utilisaient déjà beaucoup, mais l'accentue chez les seconds qui voient sa proportion augmenter au détriment des autres modes moins émetteurs).

Au sein du groupe à revenu aisé/médian, la localisation introduit une segmentation supplémentaire et importante. La centralité favorise l'allongement des distances et la portée moyenne des déplacements des personnes résidant des zones denses est supérieure d'environ $50 \%$ à celle des déplacements des personnes de même niveau de revenu résidant dans les zones périphériques et périurbaines. L'écart est surtout perceptible dans les cas du train et de l'avion, avec des rapports allant respectivement de 1 à
3 et de 1 à 4 entre les deux sous-groupes, alors que la longueur des déplacements automobiles est la même dans ces deux sous-groupes. Ces écarts entre modes n'ont pas changé au cours de la période.

Les émissions des étudiants $(3,9 \%$ de la population pour 4,9\% des émissions en 1994) ont diminué sur la période malgré l'augmentation des effectifs de cette catégorie $(4,6 \%$ de la population pour 3,8\% des émissions en 2008). En effet, si leur usage de la voiture est resté stable (un peu moins de $2800 \mathrm{~km} / \mathrm{an}$ aux deux dates), leurs recours au train et à l'avion (respectivement $1931 \mathrm{~km}$ et $2107 \mathrm{~km}$ en 1994) ont fortement diminué (respectivement -35 et $-42 \%)$.

Enfin, les scolaires du primaire et du secondaire sont beaucoup moins émetteurs que les adultes, avec $317 \mathrm{~kg}$ de $\mathrm{CO}_{2}$ émis contre 491 en moyenne sur la population en 1994. Ils parcourent un peu moins de distances que la moyenne (16\% en moins). Leurs émissions sont plus faibles du fait que leurs déplacements automobiles se font toujours en tant que passager. La segmentation par revenu reste pertinente, avec des variations de 1 à 2 selon que ces scolaires appartiennent à un ménage modeste ou à un ménage aisé, tant en termes de kilomètres parcourus que d'émissions. Surtout, ces écarts se creusent sur la période : alors que les émissions augmentent de $25 \%$ dans le cas d'enfants scolarisés appartenant à des ménages aisés, notamment du fait d'une croissance des voyages en avion, elles baissent de plus de moitié dans les milieux modestes du fait d'une baisse de $31 \%$ des distances totales parcourues par ces enfants.

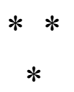

En conclusion, en matière de mobilité locale, la forte opposition entre les évolutions constatées dans les zones centrales denses et les autres zones doit être soulignée car elle apparaît transversale aux différents groupes. D'un côté, les émissions par tête des résidents des zones denses baissent de façon marquée (- 35 à - $40 \%$

1. L'échantillon des actifs modestes de périphérie et de périurbain est trop faible (369 personnes en 1994, et 285 en 2008) pour permettre de saisir finement des déplacements extrêmement peu nombreux (environ 1 par an). Des aléas d'enquête ou de redressement peuvent en conséquence affecter les résultats. 
chez les étudiants et les inactifs, - 25 à - $30 \%$ chez les actifs). Cette diminution s'explique par un abandon relatif de l'usage de l'automobile, avec une baisse du nombre de déplacements correspondants et une stabilité tant des distances parcourues par déplacement que des émissions unitaires. À l'opposé, la progression de la dépendance à l'automobile se poursuit dans les zones moins denses, avec un nombre de déplacements en voiture par personne qui se maintient mais, surtout, une croissance de leur portée et une tendance à l'individualisation de l'usage de la voiture.

L'analyse typologique révèle par ailleurs la nette progression des émissions imputables aux femmes actives. Leur nombre continue à augmenter fortement, et leurs comportements de mobilité domicile-travail rejoignent progressivement ceux de leurs homologues masculins. Par ailleurs, le renouvellement des générations consacre l'entrée de l'automobile chez les seniors. Les retraités résidant dans les centres urbains font même exception au recul constaté de la voiture dans ces zones : ils se signalent au contraire par une croissance des déplacements, des distances et des émissions liées à l'automobile. À ce niveau des déplacements locaux, cette double opposition entre centre et périphérie d'une part et entre évolutions démographiques et comportementales des différents groupes d'autre part, conduit à un renforcement de l'usage de l'automobile et à une croissance de $17 \%$ des émissions de $\mathrm{CO}_{2}$ sur la période.

La mobilité à longue distance apparaît quant à elle marquée par une progression des distances parcourues, qui se retrouve au sein de tous les groupes à l'exception notable des étudiants qui ont resserré leur budget train et avion. Au sein de cette tendance générale de hausse, une opposition se fait jour entre les retraités et le reste de la population. En effet, un renforcement sensible de leur motorisation se confirme aussi sur la longue distance. La voiture est en effet le principal vecteur de la croissance de leur mobilité et les émissions unitaires de ce mode ne diminuent pas car, comme dans le cas de la mobilité locale, les progrès technologiques sont neutralisés par la baisse tendancielle du taux d'occupation des véhicules. Pour les autres groupes, la part de l'avion dans les émissions initiales, comme son rôle dans la progression des distances parcourues, tend à favoriser une baisse des émissions dans la mesure où ses émissions unitaires diminuent de $25 \%$ au cours de la période (cela s'explique autant par des progrès technologiques que des taux de remplissages plus élevés, même si les travaux disponibles ne permettent pas d'établir des hypothèses de calcul aussi solides que pour la voiture). L'ajustement entre ces deux tendances conduit à une stabilité des émissions individuelles sur longue distance $(+2 \%)$ et donc à une augmentation des émissions globales $(+8 \%)$ imputable essentiellement à la croissance de la population.

Enfin, il reste à souligner la différence de nature entre la croissance des émissions selon que ces dernières sont liées aux mobilités quotidiennes ou aux mobilités plus exceptionnelles et plus lointaines. En effet, la croissance de la mobilité sur longue distance se trouve bien confirmée dans l'ENT, mais elle est compensée par une baisse des émissions unitaires. À l'inverse, en matière de mobilité locale, même si l'étalement urbain ne connaît plus la même vigueur que dans les années 1970 et 1980, il se poursuit toujours, avec l'allongement des distances et le nécessaire recours à l'automobile qu'il signifie. Les évolutions des comportements enregistrées dans les villes-centres ne compensent pas ce phénomène et ce sont bien, in fine, les dynamiques locales qui sont restées le principal déterminant de la progression des émissions de gaz à effet de serre liées aux mobilités individuelles au cours de ces dernières années.

\section{BIBLIOGRAPHIE}

Armoogum J., François D., Gascon M.O. et Madre J.L. (2010), "Les enquêtes nationales et locales sur la mobilité : sources et méthodes "), La revue du CGDD, pp. 207-218.

Besson D., 2008, «Consommation d'énergie : autant de dépenses en carburants qu'en énergie domestique », Insee Première $\mathrm{n}^{\circ}$ 1176. [http:// www.insee.fr/fr/ffc/ipweb/ip1176/ip1176.pdf] Diffusion de l'automobile en France : vers quels plafonds pour la motorisation et l'usage?

Collet R., Madre J.L. et Hivert L. (2012), «L'automobile en France : de la diffusion sociale 
vers la saturation?», Économie et Statistique, dans ce numéro.

Bouzouina L., Nicolas J.-P., Vanco F. (2011), «Évolution des émissions de $\mathrm{CO}_{2}$ liées aux mobilités quotidiennes : une stabilité en trompe l'œil ». Recherche Transports Sécurité, vol. 27, $\mathrm{n}^{\circ} 2$, pp. 128-139.

Cervero R. 2002, «Build environments and mode choice: toward a normative framework», Transportation Research Part D, vol. 7, $\mathrm{n}^{\circ} 4$, pp. 265-284.

Cervero R. et Kockelman K. (1997), « Travel demand and the 3Ds: density, diversity and design », Transportation Research Part D, vol. 2, $\mathrm{n}^{\circ} 3$, pp. 210-225.

Chèze B., Gastineau P. et Chevallier J. (2011), « Air traffic energy efficiency differs from place to place: analysis of historical trends by geographical zones using a macro-level methodology ", Les cahiers de l'économie, $\mathrm{n}^{\circ} 86$, pp. 1-31.

CITEPA - Centre Interprofessionnel Technique d'Etudes de la Pollution Atmosphérique (2010), Substances relatives à l'accroissement de l'effet de serre. [http://www.citepa.org]

DGAC (2010), Les émissions gazeuses liées au trafic aérien en France en 2009. Rapport interne. [http://www.developpement-durable.gouv.fr/ IMG/pdf/Emissionsn_gazeuses_Document finalV1-1.pdf]

Deloitte Consultant (2008), Efficacité énergétique et environnementale des modes de transport. Rapport pour le compte de l'ADEME, 27 p.

Dupuy G. (1999), La dépendance automobile Symptômes, analyse, diagnostic, traitement. Paris, Anthropos.

European Communities, DG VII (1999), MEET, Methodology for calculating transport emissions and energy consumption. Luxembourg, Rapport commun Inrets, AUTh, TRL, TÜV, DTU. 362 p.

Fouchier V., 1997, Les densités urbaines et le développement durable: le cas de l'Île-de-France et des villes nouvelles. Paris, Secrétariat Général du Groupe des Villes Nouvelles.

Fuglestvedt J.S., Shine K.P., Berstsen T., Cook J., Lee D.S., Skeie R.B., Velders G.J.M. et Waitz I.A. (2010), « Transport impacts on atmosphere and climate : Metrics ", Atmospheric Environment $\mathrm{n}^{\circ} 44$ (2010), pp. 4648-4677.

Gallez C. (1995), Budgets énergie environnement des déplacements (BEED) en Île-de-France. Rapport de convention ADEME-Inrets, Arcueil, Inrets, 109 p.

Gallez C. et Hivert L. (1998), BEED : mode d'emploi. Synthèse méthodologique pour les études "budget énergie environnement des déplacements". Rapport de convention ADEMEINRETS, $85 \mathrm{p}$.

Gallez C. et Orfeuil J-P. (1998) « Dis-moi où tu habites, je te dirai comment tu te déplaces », in Pumain D. et Mattei M.F. (éd.), Données urbaines, $\mathrm{n}^{\circ} 2$, Anthropos, pp. 157-164.

GIEC (2007), Bilan 2007 des changements climatiques : l'atténuation. Contribution du groupe de travail III au $4^{\text {ème }}$ rapport du GIEC. [http://www. ipcc.ch/pdf/assessment-report/ar4/wg3/ar4-wg3ts-fr.pdf]

Giuliano G. et Dargay J. (2006), « Car ownership, travel and land use: a comparison of the US and Great Britain », Transportation Research Part A, $\mathrm{n}^{\circ} 40$, pp. 106-124.

Grimal R. (2010), « Mobilité à longue distance : plus de voyages s'effectuent en train, mais les seniors restent adeptes de la voiture ", La Revue du CGDD, dossier thématique la mobilité des Français. pp. 123-150.

Hivert L., Dupont A., Quetelard B. et Merle N. (2009), Le Diagnostic Environnemental de la Mobilité 2006 Consommation énergétique, émissions de gaz à effet de serre et de polluants locaux de l'air : quel est l'impact des transports et des déplacements ? Rapport de convention ADEMEInrets-CETE NP, $149 \mathrm{p}$.

Hjorthol R.J. (2003), Gendered aspects of travel behaviour development - are the differences disappearing? Association for European Transport. $65 \mathrm{p}$.

Hourriez J.-M. et Ollier L. (1997), « Niveau de vie et taille du ménage : estimations d'une échelle d'équivalence », Économie et Statistique, n 308 309-310, pp. 65-94.

Hubert J-P. et Delisle F. (2010), « L'allongement des déplacements quotidiens contribue à l'émergence d'espaces urbains multipolaires, tandis que la mobilité baisse au centre des grandes 
agglomérations », La Revue du CGDD, dossier thématique la mobilité des Français. pp. 49-64.

IEA - International Energy Agency (2010), $\mathrm{CO}_{2}$ emissions from fuel combustion - Highlights. [http:// www.iea.org/co2highlights/co2highlights.pdf]

Joly M., Morency C. et Bonnel P. (2009), "Motorisation et localisation: quels effets sur le choix modal ? », Les Cahiers Scientifiques $d u$ Transport $\mathrm{n}^{\circ} 55$, pp. 97-125.

Kaufmann V. et Flamm M. (2002), Famille, temps et mobilité : état de l'art et tour d'horizon des innovations. LASUR, École Polytechnique de Lausanne, rapport de recherche pour la CNAF et l'Institut pour la Ville en Mouvement. 62 p.

Lipps O. et Kunert U. (2005), « Measuring and explaining the increase of travel distance: a multilevel analysis using repeated cross sectional travel surveys », DIW Berlin, Discussion Papers $\mathrm{n}^{\circ} 492$, $25 \mathrm{p}$.

Lleras G., Simma A., Ben-Akiva M., Shaefer A., Axhausen K. et Furutani T. (2003), «Fundamental relationships specifying travel behavior - an international travel survey comparison », TRB 2003 Annual Meeting CD-Rom, 19 p.

Longuar Z., Nicolas JP. et Verry D. (2012), Méthodologie de calcul des émissions de $\mathrm{CO}_{2}$ dans l'Enquête Nationale Transports et Déplacements de 2008. Lyon, Rapport LET-CERTU. À paraître.

Madre J.-L. (1997), "L'enquête transports ", RTS n56, Spécial enquête Transports et Communications, pp. 6-8.

Massot M.-H., Orfeuil J.-P. et Bellanger F. (2000), Éléments pour une prospective de la mobilité. Rapport CRETEIL, Université Paris XII. 65 p.

Mercado R. et Miller E. (2010), « Investigating changes in travel behaviour of the older population in the greater Toronto and Hamilton area - 1986$2006 », 12^{\text {th }}$ World Conference on Transport Research, July 11-15, Lisbon, Portugal, 15 p.

Mézière N. (2004), Typologie des comportements de mobilité. Lyon, rapport de stage ENSAE-LET.

Morency C., Paez A., Roorda M., Mercad R. et Farber S. (2011), « Distanced traveled in three Canadian cities: spatial analysis from the perspective of vulnerable population segments », Journal of Transport Geography n ${ }^{\circ}$ 19, pp. 39-50.
Nicolas J.-P. et David D. (2009), « Passenger transport and $\mathrm{CO}_{2}$ emissions: What does the French transport survey tell us? », Atmospheric Environment $\mathrm{n}^{\circ} 43$ (2009), pp. 1015-1020.

Nicolas J.-P., Pochet P., Poimboeuf H. (2002), «Mobilité urbaine et développement durable: quels outils de mesure pour quels enjeux?», Cahiers Scientifiques du Transport, $\mathrm{n}^{\circ} 41, \mathrm{pp}$. 53-76.

Ntziachristos L., Samaras Z., Kouridis C., Hassel D, McCrae I., Hickman J., Zierock K.H., Keller M., André M., Gorissen N., Dilara P., Boulter P., Joumard R., Rijkeboer R., Geivanidis S. et Hausberger S. (2010), EMEP/ CORINAIR Atmospheric Emissions Inventory Guidebook on exhaust emissions from road transport COPERT 4, Methodology for the calculation of exhaust emissions, European Environment Agency Report, 129 p. http://www.emisia.com/ copert/

Orfeuil J.-P. (1984), « Les budgets énergie-transport : un concept, une pratique, des résultats $»$. $R T S, \mathrm{n}^{\circ} 2$, pp. 23-29.

Orfeuil J.-P. (2000), « L'évolution de la mobilité quotidienne : comprendre les dynamiques, éclairer les controverses ». Synthèse Inrets $n^{\circ} 37$.

Orfeuil J.-P. (2002), «État des lieux des recherches sur la mobilité quotidienne en France », in Lévy J-P. et Dureau F. (eds), L'accès à la ville. Les mobilités spatiales en question. Paris, L'Harmattan. pp. 65-93.

Orfeuil J-P. et Soleyret D. (2002), « Quelles interactions entre les marchés de la mobilité à courte et à longue distance? ». Recherche Transport Sécurité nº 76, pp. 208-221.

Paulo C. (2006), Inégalités de mobilités : disparités de revenus, hétérogénéité des effets. Thèse de doctorat en sciences économiques, Université Lumière Lyon 2.

Paulo, C. (2006), «L'impact du revenu sur les mobilités : une mesure à différentes échelles spatiales et temporelles », XLII ${ }^{\circ}$ Colloque de l'ASR$D L F$, Sfax, Tunisie, 4-6 sept. 2006. 20 p.

Pouyanne G. (2004), Forme urbaine et mobilité quotidienne. Université Montesquieu Bordeaux, thèse de doctorat en sciences économiques.

Quételard B. (2010), «Se rendre au travail ou faire ses courses motive toujours un déplacement 
quotidien sur deux », La Revue du CGDD, dossier thématique La mobilité des Français. pp. 25-48.

Rajamani J., Bhat C., Handy S., Knaap G. et Song Y. (2003), «Assessing the impact of urban form measures in nonwork trip mode choice after controlling for demographic and level-of-service effects », Journal of the Transport Research Board, vol. 1831, pp. 158-165.

Raux C., Traisnel J.P., Nicolas J.P. et Delvert K. (2005), Bilans énergétiques Transport-Habitat et méthodologie BETEL. Lyon, LET, projet ETHEL, rapport R2, Action concertée CNRS-Ministère de la Recherche. 138 p.

Raux C., Traisnel J.P., Pochet, P., Maïzia M., Croissant Y., Bagard V. et Péguy P.Y. (2006), Analyse et modélisation des comportements transports-habitat-localisations. Lyon, LET, projet ETHEL, rapport R3, Action concertée CNRS - Ministère de la Recherche. 138 p.
Rouquette C. (2001), « Départs en vacances : la persistance des inégalités », Économie et Statistique, $\mathrm{n}^{\circ} 345$, pp. 33-53.

Timmermans H., van der Waerden P., Alves M., Polak J., Ellis S., Harvey A., Kurose S. et Zandee D. (2003), « Spatial context and the complexity of daily travel patterns: an international comparison ", Journal of Transport Geography $\mathrm{n}^{\circ} 11$, pp. 37-46.

Vanco F. (2011), Formes urbaines et durabilité du système de transports - une approche par les coûts du la mobilité urbaine des ménages sur l'agglomération lyonnaise. Université Lumière Lyon 2 , thèse de doctorat en sciences économiques.

Viard J. (2002), « Temps libre, loisirs, vacances et art de vivre : le triangle des Bermudes des sociétés modernes », in J. Viard, F. Potier et J.-D. Urbain. La France des temps libres et des vacances. Éditions de l'Aube, 2002, pp. 3-22. 
